\title{
Envelopes of one-parameter families of framed curves in the Euclidean space
}

\author{
Donghe Pei, Masatomo Takahashi, and Haiou Yu®
}

\begin{abstract}
As a one-parameter family of singular space curves, we consider a one-parameter family of framed curves in the Euclidean space. Then we define an envelope for a one-parameter family of framed curves and investigate properties of envelopes. Especially, we concentrate on oneparameter families of framed curves in the Euclidean 3-space. As applications, we give relations among envelopes of one-parameter families of framed space curves, one-parameter families of Legendre curves and oneparameter families of spherical Legendre curves, respectively.
\end{abstract}

Mathematics Subject Classification. 58K05, 53A40, 57R45.

Keywords. Envelope, one-parameter families of framed curves, singularity.

\section{Introduction}

For regular plane curves, envelopes are classical objects in differential geometry $[1,2,6,7,10,12]$. An envelope of a family of curves is a new curve which is tangent to curves of the given family. An envelope of a family of submanifolds in the Euclidean space were studied in $[3,15]$. Using the notion of stability for germs of family, they investigate the singularities of the envelope of the submanifolds in the Euclidean space via Legendrian singularity theory. However, by using implicit functions, the definition and calculation of the envelope of space curves are complicated.

On the other hand, if we look at the classical concept of envelope we see that the envelope is the set of characteristic points and avoiding singularities of the elements of the family. Moreover, for singular space curves, the classical definitions of envelopes are vague. In [14], the second author clarified the definition of the envelope for families of singular plane curves. In [11], we clarified the 
definition of the envelope for families of singular spherical curves. In this paper we pursue second author's method and treat the envelope of smooth space curves with singular points. We would like to define an envelope of parametric space curves with singular points in the Euclidean space. As a singular curve, we consider a framed curve in the Euclidean space. A framed curve in the Euclidean space is a smooth curve with a moving frame (cf. [8]). It is a generalization of a regular curve with linear independent condition, a Legendre curve in the unit tangent bundle over the Euclidean plane and a Legendre curve in the unit spherical bundle over the unit sphere.

In Sect. 2, we consider one-parameter families of framed curves in the Euclidean space. We give a moving frame and the curvature of a one-parameter family of framed curves. Then we give the existence and uniqueness theorems in terms of the curvatures of the one-parameter families of framed curves. In Sect. 3, we define an envelope of a one-parameter family of framed curves in the Euclidean space. We obtain that the envelope is also a framed curve. As a main result, we give a necessary and sufficient condition that a one-parameter family of framed curves has an envelope (Theorem 3.3). In Sect. 4, we focus on the framed curves in the Euclidean 3-space. The theory of one-parameter families of framed curves relate to the theory of surfaces with singular points (cf. $[4,5])$. The envelope is independent of rotated frames and reflected frames of the framed curves. We also define a parallel curve of a one-parameter family of framed space curves. The parallel curve is also a one-parameter family of framed curves. Then the envelope of parallel curves and a parallel curve of the envelope of a one-parameter family of framed curves coincide (Proposition 4.7). In Sect. 5, as applications, we give relations among envelopes of one-parameter families of framed space curves, one-parameter families of Legendre curves in the unit tangent bundle over the Euclidean plane and one-parameter families of Legendre curves in the unit spherical bundle over the unit sphere, respectively. In Sect. 6, we give concrete examples of envelopes of one-parameter families of framed curves.

All maps and manifolds considered here are differentiable of class $C^{\infty}$.

\section{Framed curves and one-parameter families of framed curves in the Euclidean space}

We first recall some definitions and results of framed curves in the Euclidean space. For more details see [8].

Let $\mathbb{R}^{n}$ be the $n$-dimensional Euclidean space equipped with the inner product $\boldsymbol{a} \cdot \boldsymbol{b}=\sum_{i=1}^{n} a_{i} b_{i}$, where $\boldsymbol{a}=\left(a_{1}, \ldots, a_{n}\right)$ and $\boldsymbol{b}=\left(b_{1}, \ldots, b_{n}\right)$. We denote the norm of $\boldsymbol{a}$ by $|\boldsymbol{a}|=\sqrt{\boldsymbol{a} \cdot \boldsymbol{a}}$. Let $\boldsymbol{a}_{1}, \ldots, \boldsymbol{a}_{n-1} \in \mathbb{R}^{n}$ be vectors $\boldsymbol{a}_{i}=\left(a_{i 1}, \ldots, a_{i n}\right)$ for $i=1, \ldots, n-1$. The vector product is given by 


$$
\boldsymbol{a}_{1} \times \cdots \times \boldsymbol{a}_{n-1}=\operatorname{det}\left(\begin{array}{ccc}
a_{11} & \cdots & a_{1 n} \\
\vdots & \ddots & \vdots \\
a_{n-11} & \cdots & a_{n-1 n} \\
\boldsymbol{e}_{1} & \cdots & \boldsymbol{e}_{n}
\end{array}\right)=\sum_{i=1}^{n} \operatorname{det}\left(\boldsymbol{a}_{1}, \ldots, \boldsymbol{a}_{n-1}, \boldsymbol{e}_{i}\right) \boldsymbol{e}_{i}
$$

where $\left\{\boldsymbol{e}_{1}, \ldots, \boldsymbol{e}_{n}\right\}$ is the canonical basis of $\mathbb{R}^{n}$. Then we have $\left(\boldsymbol{a}_{1} \times \cdots \times\right.$ $\left.\boldsymbol{a}_{n-1}\right) \cdot \boldsymbol{a}_{i}=0$ for $i=1, \ldots, n-1$. Note that for the case of $n=3$,

$$
\boldsymbol{a}_{1} \times \boldsymbol{a}_{2}=\operatorname{det}\left(\begin{array}{ccc}
a_{11} & a_{12} & a_{13} \\
a_{21} & a_{22} & a_{23} \\
\boldsymbol{e}_{1} & \boldsymbol{e}_{2} & \boldsymbol{e}_{3}
\end{array}\right)=\operatorname{det}\left(\begin{array}{ccc}
\boldsymbol{e}_{1} & \boldsymbol{e}_{2} & \boldsymbol{e}_{3} \\
a_{11} & a_{12} & a_{13} \\
a_{21} & a_{22} & a_{23}
\end{array}\right) .
$$

Let $S^{n-1}=\left\{\left(x_{1}, \ldots, x_{n}\right) \in \mathbb{R}^{n} \mid x_{1}^{2}+\cdots+x_{n}^{2}=1\right\}$ be the unit sphere. We denote the set $\Delta_{n-1}$,

$$
\begin{aligned}
\Delta_{n-1} & =\left\{\boldsymbol{\nu}=\left(\nu_{1}, \ldots, \nu_{n-1}\right) \in \mathbb{R}^{n} \times \cdots \times \mathbb{R}^{n} \mid \nu_{i} \cdot \nu_{j}=\delta_{i j}, i, j=1, \ldots, n-1\right\} \\
& =\left\{\boldsymbol{\nu}=\left(\nu_{1}, \ldots, \nu_{n-1}\right) \in S^{n-1} \times \cdots \times S^{n-1} \mid \nu_{i} \cdot \nu_{j}=0, i \neq j, i, j=1, \ldots, n-1\right\} .
\end{aligned}
$$

Then $\Delta_{n-1}$ is a $n(n-1) / 2$-dimensional smooth manifold.

Let $I$ be an interval of $\mathbb{R}$.

Definition 2.1. Let $(\gamma, \boldsymbol{\nu}): I \rightarrow \mathbb{R}^{n} \times \Delta_{n-1}$ be a smooth mapping. We say that $(\gamma, \boldsymbol{\nu})$ is a framed curve if $\dot{\gamma}(t) \cdot \nu_{i}(t)=0$ for all $t \in I$ and $i=1, \ldots, n-1$, where $\boldsymbol{\nu}=\left(\nu_{1}, \ldots, \nu_{n-1}\right)$.

We define $\boldsymbol{\mu}: I \rightarrow S^{n-1}$ by $\boldsymbol{\mu}(t)=\nu_{1}(t) \times \cdots \times \nu_{n-1}(t)$. By definition, $(\boldsymbol{\nu}(t), \boldsymbol{\mu}(t)) \in \Delta_{n}$ and $\{\boldsymbol{\nu}(t), \boldsymbol{\mu}(t)\}$ is a moving frame along $\gamma(t)$. Then we have the Frenet type formula.

$$
\left(\begin{array}{c}
\dot{\boldsymbol{\nu}}(t) \\
\dot{\boldsymbol{\mu}}(t)
\end{array}\right)=A(t)\left(\begin{array}{c}
\boldsymbol{\nu}(t) \\
\boldsymbol{\mu}(t)
\end{array}\right), \dot{\gamma}(t)=\alpha(t) \boldsymbol{\mu}(t)
$$

where $A(t)=\left(\alpha_{i j}(t)\right) \in \mathfrak{o}(n), i, j=1, \ldots, n, \mathfrak{o}(n)$ is the set of $n \times n$ alternative matrices and $\alpha: I \rightarrow \mathbb{R}$ is a smooth function. We say that the mapping $\left(\alpha_{i j}, \alpha\right)$ is the curvature of the framed curve $(\gamma, \boldsymbol{\nu})$.

Definition 2.2. Let $(\gamma, \boldsymbol{\nu})$ and $(\widetilde{\gamma}, \widetilde{\boldsymbol{\nu}}): I \rightarrow \mathbb{R}^{n} \times \Delta_{n-1}$ be framed curves. We say that $(\gamma, \boldsymbol{\nu})$ and $(\widetilde{\gamma}, \widetilde{\boldsymbol{\nu}})$ are congruent as framed curves if there exist a special orthogonal matrix $X \in S O(n)$ and a constant vector $\boldsymbol{x} \in \mathbb{R}^{n}$ such that $\widetilde{\gamma}(t)=X(\gamma(t))+\boldsymbol{x}$ and $\widetilde{\boldsymbol{\nu}}(t)=X(\boldsymbol{\nu}(t))$ for all $t \in I$.

Then we have the existence and uniqueness theorems in terms of the curvatures of the framed curves.

Theorem 2.3 (Existence Theorem for framed curves [8]). Let $\left(\alpha_{i j}, \alpha\right): I \rightarrow$ $\mathfrak{o}(n) \times \mathbb{R}$ be a smooth mapping. Then there exists a framed curve $(\gamma, \boldsymbol{\nu}): I \rightarrow$ $\mathbb{R}^{n} \times \Delta_{n-1}$ whose associated curvature is given by $\left(\alpha_{i j}, \alpha\right)$. 
Theorem 2.4 (Uniqueness Theorem for framed curves [8]). Let $(\gamma, \boldsymbol{\nu})$ and $(\widetilde{\gamma}, \widetilde{\boldsymbol{\nu}}): I \rightarrow \mathbb{R}^{n} \times \Delta_{n-1}$ be framed curves with the curvatures $\left(\alpha_{i j}, \alpha\right)$ and $\left(\widetilde{\alpha}_{i j}, \widetilde{\alpha}\right)$ respectively. Then $(\gamma, \boldsymbol{\nu})$ and $(\widetilde{\gamma}, \widetilde{\boldsymbol{\nu}})$ are congruent as framed curves if and only if $\left(\alpha_{i j}, \alpha\right)$ and $\left(\widetilde{\alpha}_{i j}, \widetilde{\alpha}\right)$ coincide.

Next, we consider one-parameter families of framed curves in the Euclidean space. Let $\Lambda$ be open interval of $\mathbb{R}$.

Definition 2.5. Let $(\gamma, \boldsymbol{\nu}): I \times \Lambda \rightarrow \mathbb{R}^{n} \times \Delta_{n-1}$ be a smooth mapping. We say that $(\gamma, \boldsymbol{\nu})$ is a one-parameter family of framed curves if $\gamma_{t}(t, \lambda) \cdot \nu_{i}(t, \lambda)=0$ for all $(t, \lambda) \in I \times \Lambda$ and $i=1, \ldots, n-1$, where $\boldsymbol{\nu}=\left(\nu_{1}, \ldots, \nu_{n-1}\right)$.

By definition, $(\gamma(\cdot, \lambda), \boldsymbol{\nu}(\cdot, \lambda)): I \rightarrow \mathbb{R}^{n} \times \Delta_{n-1}$ is a framed curve for each fixed parameter $\lambda \in \Lambda$. It is easy to see that $(-\gamma, \boldsymbol{\nu})$ and $(\gamma,-\boldsymbol{\nu})$ are also one-parameter families of framed curves.

We define $\boldsymbol{\mu}(t, \lambda)=\nu_{1}(t, \lambda) \times \cdots \times \nu_{n-1}(t, \lambda)$. Then $\{\boldsymbol{\nu}(t, \lambda), \boldsymbol{\mu}(t, \lambda)\}$ is a moving frame along $\gamma(t, \lambda)$ and we have the Frenet type formula.

$$
\begin{gathered}
\left(\begin{array}{l}
\boldsymbol{\nu}_{t}(t, \lambda) \\
\boldsymbol{\mu}_{t}(t, \lambda)
\end{array}\right)=A(t, \lambda)\left(\begin{array}{l}
\boldsymbol{\nu}(t, \lambda) \\
\boldsymbol{\mu}(t, \lambda)
\end{array}\right), \quad\left(\begin{array}{l}
\boldsymbol{\nu}_{\lambda}(t, \lambda) \\
\boldsymbol{\mu}_{\lambda}(t, \lambda)
\end{array}\right)=B(t, \lambda)\left(\begin{array}{c}
\boldsymbol{\nu}(t, \lambda) \\
\boldsymbol{\mu}(t, \lambda)
\end{array}\right), \\
\gamma_{t}(t, \lambda)=\alpha(t, \lambda) \boldsymbol{\mu}(t, \lambda), \gamma_{\lambda}(t, \lambda)=P(t, \lambda)\left(\begin{array}{c}
\boldsymbol{\nu}(t, \lambda) \\
\boldsymbol{\mu}(t, \lambda)
\end{array}\right),
\end{gathered}
$$

where $A(t, \lambda)=\left(\alpha_{i j}(t, \lambda)\right), B(t, \lambda)=\left(\beta_{i j}(t, \lambda)\right) \in \mathfrak{o}(n), i, j=1, \ldots, n, \alpha$ : $I \times \Lambda \rightarrow \mathbb{R}$ is a smooth function and $P: I \times \Lambda \rightarrow \mathbb{R}^{n}, P(t, \lambda)=\left(P_{1}(t, \lambda), \ldots\right.$, $\left.P_{n}(t, \lambda)\right)$ is a smooth mapping.

By $\gamma_{t \lambda}(t, \lambda)=\gamma_{\lambda t}(t, \lambda), \nu_{t \lambda}(t, \lambda)=\nu_{\lambda t}(t, \lambda)$ and $\boldsymbol{\mu}_{t \lambda}(t, \lambda)=\boldsymbol{\mu}_{\lambda t}(t, \lambda)$, we have the integrability condition

$$
\begin{aligned}
& \left(\alpha_{i j}(t, \lambda)\right)_{\lambda}+\left(\alpha_{i j}(t, \lambda)\right)\left(\beta_{i j}(t, \lambda)\right)=\left(\beta_{i j}(t, \lambda)\right)_{t}+\left(\beta_{i j}(t, \lambda)\right)\left(\alpha_{i j}(t, \lambda)\right), \\
& \alpha(t, \lambda) \beta_{n i}(t, \lambda)=\left(P_{i}\right)_{t}(t, \lambda)+\sum_{j=1}^{n} P_{j}(t, \lambda) \alpha_{j i}(t, \lambda),(i=1, \ldots, n-1), \\
& \alpha_{\lambda}(t, \lambda)+\alpha(t, \lambda) \beta_{n n}(t, \lambda)=\left(P_{n}\right)_{t}(t, \lambda)+\sum_{j=1}^{n} P_{j}(t, \lambda) \alpha_{j n}(t, \lambda)
\end{aligned}
$$

for all $(t, \lambda) \in \mathrm{I} \times \Lambda$. We call the mapping $\left(\alpha_{i j}, \beta_{i j}, \alpha, P_{1}, \ldots, P_{n}\right)$ with the integrability condition (2.1) the curvature of the one-parameter family of framed curves $(\gamma, \boldsymbol{\nu})$.

Definition 2.6. Let $(\gamma, \boldsymbol{\nu})$ and $(\widetilde{\gamma}, \widetilde{\boldsymbol{\nu}}): I \times \Lambda \rightarrow \mathbb{R}^{n} \times \Delta_{n-1}$ be one-parameter families of framed curves. We say that $(\gamma, \boldsymbol{\nu})$ and $(\widetilde{\gamma}, \widetilde{\boldsymbol{\nu}})$ are congruent as oneparameter families of framed curves if there exist a special orthogonal matrix $X \in S O(n)$ and a constant vector $\boldsymbol{x} \in \mathbb{R}^{n}$ such that $\widetilde{\gamma}(t, \lambda)=X(\gamma(t, \lambda))+\boldsymbol{x}$ and $\widetilde{\boldsymbol{\nu}}(t, \lambda)=X(\boldsymbol{\nu}(t, \lambda))$ for all $(t, \lambda) \in I \times \Lambda$.

We give the existence and uniqueness theorems in terms of the curvatures of the one-parameter families of framed curves. 
Theorem 2.7 (Existence Theorem for one-parameter families of framed curves). Let $\left(\alpha_{i j}, \beta_{i j}, \alpha, P_{1}, \ldots, P_{n}\right): I \times \Lambda \rightarrow \mathfrak{o}(n) \times \mathfrak{o}(n) \times \mathbb{R}^{n+1}$ be a smooth mapping with the integrability condition. Then there exists a one-parameter family of framed curves $(\gamma, \boldsymbol{\nu}): I \times \Lambda \rightarrow \mathbb{R}^{n} \times \Delta_{n-1}$ whose associated curvature is given by $\left(\alpha_{i j}, \beta_{i j}, \alpha, P_{1}, \ldots, P_{n}\right)$.

Proof. We denote that $M(n)$ is the set of $n \times n$ matrices and $I_{n}$ is the identity matrix. Choose any fixed value $\left(t_{0}, \lambda_{0}\right) \in I \times \Lambda$. We consider an initial value problem,

$$
F_{t}(t, \lambda)=A(t, \lambda) F(t, \lambda), F_{\lambda}(t, \lambda)=B(t, \lambda) F(t, \lambda), F\left(t_{0}, \lambda_{0}\right)=I_{n},
$$

where $F(t, \lambda) \in M(n)$ and $A(t, \lambda)=\left(\alpha_{i j}(t, \lambda)\right), B(t, \lambda)=\left(\beta_{i j}(t, \lambda)\right) \in \mathfrak{o}(n)$ for $i, j=1, \ldots, n$. By

$$
\begin{aligned}
& F_{t \lambda}=A_{\lambda} F+A F_{\lambda}=A_{\lambda} F+A B F=\left(A_{\lambda}+A B\right) F, \\
& F_{\lambda t}=B_{t} F+B F_{t}=B_{t} F+B A F=\left(B_{t}+B A\right) F .
\end{aligned}
$$

and the integrability condition $A_{\lambda}+A B=B_{t}+B A$, we have $F_{t \lambda}=F_{\lambda t}$. Since $I \times \Lambda$ is simply connected, there exists a solution $F(t, \lambda)$. Since $A(t, \lambda), B(t, \lambda) \in$ $\mathfrak{o}(n)$,

$$
\begin{aligned}
& \frac{\partial}{\partial t}\left(F(t, \lambda){ }^{t} F(t, \lambda)\right)=F(t, \lambda) \frac{\partial}{\partial t}{ }^{t} F(t, \lambda)+\frac{\partial}{\partial t} F(t, \lambda){ }^{t} F(t, \lambda) \\
& \quad=F(t, \lambda)\left({ }^{t} A(t, \lambda)+A(t, \lambda)\right){ }^{t} F(t, \lambda)=O, \\
& \frac{\partial}{\partial \lambda}\left(F(t, \lambda){ }^{t} F(t, \lambda)\right)=F(t, \lambda) \frac{\partial}{\partial \lambda}{ }^{t} F(t, \lambda)+\frac{\partial}{\partial \lambda} F(t, \lambda){ }^{t} F(t, \lambda) \\
& \quad=F(t, \lambda)\left({ }^{t} B(t, \lambda)+B(t, \lambda)\right)^{t} F(t, \lambda)=O .
\end{aligned}
$$

It follows that $F(t, \lambda){ }^{t} F(t, \lambda)$ is constant. Therefore, we have

$$
F(t, \lambda)^{t} F(t, \lambda)=F\left(t_{0}, \lambda_{0}\right)^{t} F\left(t_{0}, \lambda_{0}\right)=I_{n} .
$$

Let $F(t, \lambda)={ }^{t}\left(\nu_{1}(t, \lambda), \ldots, \nu_{n-1}(t, \lambda), \boldsymbol{\mu}(t, \lambda)\right)$. Since

$$
(\partial / \partial t)(\operatorname{det} F(t, \lambda))=0,(\partial / \partial \lambda)(\operatorname{det} F(t, \lambda))=0
$$

we have

$$
\operatorname{det} F(t, \lambda)=\operatorname{det} F\left(t_{0}, \lambda_{0}\right)=\operatorname{det} I_{n}=1 .
$$

Hence $F(t, \lambda)$ is a special orthogonal matrix. Then $\boldsymbol{\mu}(t, \lambda)=\nu_{1}(t, \lambda) \times \cdots \times$ $\nu_{n-1}(t, \lambda)$. Next we consider differential equations

$$
\gamma_{t}=\alpha \boldsymbol{\mu}, \gamma_{\lambda}=P_{1} \nu_{1}+\cdots+P_{n-1} \nu_{n-1}+P_{n} \boldsymbol{\mu} .
$$

By the integrability condition, we have $\gamma_{t \lambda}(t, \lambda)=\gamma_{\lambda t}(t, \lambda)$ for all $(t, \lambda) \in I \times \Lambda$. Then there exists a solution $\gamma(t, \lambda)$. It follows that $(\gamma, \boldsymbol{\nu}): I \times \Lambda \rightarrow \mathbb{R}^{n} \times \Delta_{n-1}$ is a one-parameter family of framed curves whose associated curvature is given by $\left(\alpha_{i j}, \beta_{i j}, \alpha, P_{1}, \ldots, P_{n}\right)$.

Lemma 2.8. If $\left(\alpha_{i j}, \beta_{i j}, \alpha, P_{1}, \ldots, P_{n}\right)(t, \lambda)=\left(\widetilde{\alpha}_{i j}, \widetilde{\beta}_{i j}, \widetilde{\alpha}, \widetilde{P}_{1}, \ldots, \widetilde{P}_{n}\right)(t, \lambda)$ and $(\gamma, \boldsymbol{\nu})\left(t_{0}, \lambda_{0}\right)=(\widetilde{\gamma}, \widetilde{\boldsymbol{\nu}})\left(t_{0}, \lambda_{0}\right)$ for some point $\left(t_{0}, \lambda_{0}\right) \in I \times \Lambda$, then $(\gamma, \boldsymbol{\nu})=(\widetilde{\gamma}, \widetilde{\boldsymbol{\nu}})$. 
Proof. We put $\nu_{n}(t, \lambda)=\boldsymbol{\mu}(t, \lambda)$ and $\widetilde{\nu}_{n}(t, \lambda)=\widetilde{\boldsymbol{\mu}}(t, \lambda)$. We define a smooth function $f: I \times \Lambda \rightarrow \mathbb{R}$ by

$$
f(t, \lambda)=\sum_{i=1}^{n} \nu_{i}(t, \lambda) \cdot \widetilde{\nu}_{i}(t, \lambda) .
$$

By $\boldsymbol{\nu}\left(t_{0}, \lambda_{0}\right)=\widetilde{\boldsymbol{\nu}}\left(t_{0}, \lambda_{0}\right)$, we have $\nu_{i}\left(t_{0}, \lambda_{0}\right)=\widetilde{\nu}_{i}\left(t_{0}, \lambda_{0}\right)(i=1, \ldots, n)$ and hence $f\left(t_{0}, \lambda_{0}\right)=n$. Since $\alpha_{i j}(t, \lambda)=\widetilde{\alpha}_{i j}(t, \lambda)$ and $\alpha_{i j}(t, \lambda)=-\alpha_{j i}(t, \lambda)$, we have

$$
\begin{aligned}
f_{t}(t, \lambda) & =\sum_{i=1}^{n}\left(\nu_{i_{t}}(t, \lambda) \cdot \widetilde{\nu}_{i}(t, \lambda)+\nu_{i}(t, \lambda) \cdot\left(\widetilde{\nu}_{i}\right)_{t}(t, \lambda)\right) \\
& =\sum_{i=1}^{n}\left\{\left(\sum_{j=1}^{n} \alpha_{i j}(t, \lambda) \nu_{j}(t, \lambda)\right) \cdot \widetilde{\nu}_{i}(t, \lambda)+\nu_{i}(t, \lambda) \cdot\left(\sum_{j=1}^{n} \widetilde{\alpha}_{i j}(t, \lambda) \widetilde{\nu}_{j}\right)\right\} \\
& =2 \sum_{i=1}^{n} \sum_{j=1}^{n}\left(\alpha_{i j}(t, \lambda)+\alpha_{j i}(t, \lambda)\right) \nu_{i}(t, \lambda) \cdot \widetilde{\nu}_{j}(t, \lambda)=0 .
\end{aligned}
$$

In the same way, we have $f_{\lambda}(t, \lambda)=0$. It follows that $f$ is constant with value $n$. By the Cauchy-Schwarz inequality, we have

$$
\nu_{i}(t, \lambda) \cdot \widetilde{\nu}_{i}(t, \lambda) \leqslant\left|\nu_{i}(t, \lambda)\right|\left|\widetilde{\nu}_{i}(t, \lambda)\right|=1
$$

for each $i=1, \ldots, n$. If one of these inequalities is strict, the value of $f(t, \lambda)$ would be less than $n$. Therefore, these inequalities are equalities, that is, $\nu_{i}(t, \lambda) \cdot \widetilde{\nu}_{i}(t, \lambda)=1$. Then we have $\left|\nu_{i}(t, \lambda)-\widetilde{\nu}_{i}(t, \lambda)\right|^{2}=0$ and hence $\nu_{i}(t, \lambda)=$ $\widetilde{\nu}_{i}(t, \lambda)$ for all $(t, \lambda) \in I \times \Lambda$ and $i=1, \ldots, n$. Since $\gamma_{t}(t, \lambda)=\alpha(t, \lambda) \boldsymbol{\mu}(t, \lambda)$, $\widetilde{\gamma}_{t}(t, \lambda)=\widetilde{\alpha}(t, \lambda) \widetilde{\boldsymbol{\mu}}(t, \lambda), \gamma_{\lambda}(t, \lambda)=P_{1}(t, \lambda) \nu_{1}(t, \lambda)+\cdots+P_{n-1}(t, \lambda) \nu_{n-1}(t, \lambda)+$ $P_{n}(t, \lambda) \boldsymbol{\mu}(t, \lambda), \widetilde{\gamma}_{\lambda}(t, \lambda)=\widetilde{P}_{1}(t, \lambda) \widetilde{\nu}_{1}(t, \lambda)+\cdots+\widetilde{P}_{n-1}(t, \lambda) \widetilde{\nu}_{n-1}(t, \lambda)+\widetilde{P}_{n}(t, \lambda)$ $\widetilde{\boldsymbol{\mu}}(t, \lambda)$, and the assumptions $\alpha(t, \lambda)=\widetilde{\alpha}(t, \lambda), P_{i}(t, \lambda)=\widetilde{P}_{i}(t, \lambda)(i=1, \ldots, n)$, we have

$$
(\gamma(t, \lambda)-\widetilde{\gamma}(t, \lambda))_{t}=0,(\gamma(t, \lambda)-\widetilde{\gamma}(t, \lambda))_{\lambda}=0 .
$$

It follows that $\gamma(t, \lambda)-\widetilde{\gamma}(t, \lambda)$ is constant. By the condition $\gamma\left(t_{0}, \lambda_{0}\right)=\widetilde{\gamma}\left(t_{0}, \lambda_{0}\right)$, we have $\gamma(t, \lambda)=\widetilde{\gamma}(t, \lambda)$ for all $(t, \lambda) \in I \times \Lambda$.

Theorem 2.9 (Uniqueness Theorem for one-parameter families of framed curves). Let $(\gamma, \boldsymbol{\nu})$ and $(\widetilde{\gamma}, \widetilde{\boldsymbol{\nu}}): I \times \Lambda \rightarrow \mathbb{R}^{n} \times \Delta_{n-1}$ be one-parameter families of framed curves with the curvatures $\left(\alpha_{i j}, \beta_{i j}, \alpha, P_{1}, \ldots, P_{n}\right)$ and $\left(\widetilde{\alpha}_{i j}, \widetilde{\beta}_{i j}, \widetilde{\alpha}, \widetilde{P}_{1}\right.$, $\left.\ldots, \widetilde{P}_{n}\right)$, respectively. Then $(\gamma, \boldsymbol{\nu})$ and $(\widetilde{\gamma}, \widetilde{\boldsymbol{\nu}})$ are congruent as one-parameter families of framed curves if and only if $\left(\alpha_{i j}, \beta_{i j}, \alpha, P_{1}, \ldots, P_{n}\right)$ and $\left(\widetilde{\alpha}_{i j}, \widetilde{\beta}_{i j}, \widetilde{\alpha}\right.$, $\left.\widetilde{P}_{1}, \ldots, \widetilde{P}_{n}\right)$ coincide.

Proof. Suppose that $(\gamma, \boldsymbol{\nu})$ and $(\widetilde{\gamma}, \widetilde{\boldsymbol{\nu}})$ are congruent as one-parameter families of framed curves, there exist a matrix $X \in S O(n)$ and a constant vector $\boldsymbol{x} \in \mathbb{R}^{n}$ such that

$$
\widetilde{\gamma}(t, \lambda)=X(\gamma(t, \lambda))+\boldsymbol{x}, \widetilde{\boldsymbol{\nu}}(t, \lambda)=X(\boldsymbol{\nu}(t, \lambda))
$$


for all $(t, \lambda) \in I \times \Lambda$. Since the definition of $\boldsymbol{\mu}$, we have $\widetilde{\boldsymbol{\mu}}(t, \lambda)=X(\boldsymbol{\mu}(t, \lambda))$ for all $(t, \lambda) \in I \times \Lambda$. By a direct calculation, we have

$$
\begin{aligned}
\widetilde{\alpha}_{i j}(t, \lambda) & =\left(\widetilde{\nu}_{i}\right)_{t}(t, \lambda) \cdot \widetilde{\nu}_{j}(t, \lambda) \\
& =X\left(\left(\nu_{i}\right)_{t}(t, \lambda)\right) \cdot X\left(\nu_{j}(t, \lambda)\right)=\left(\nu_{i}\right)_{t}(t, \lambda) \cdot \nu_{j}(t, \lambda)=\alpha_{i j}(t, \lambda), \\
\widetilde{\beta}_{i j}(t, \lambda) & =\left(\widetilde{\nu}_{i}\right)_{\lambda}(t, \lambda) \cdot \widetilde{\nu}_{j}(t, \lambda) \\
& =X\left(\left(\nu_{i}\right)_{\lambda}(t, \lambda)\right) \cdot X\left(\nu_{j}(t, \lambda)\right)=\left(\nu_{i}\right)_{\lambda}(t, \lambda) \cdot \nu_{j}(t, \lambda)=\beta_{i j}(t, \lambda), \\
\widetilde{\alpha}(t, \lambda) & =\widetilde{\gamma}_{t}(t, \lambda) \cdot \widetilde{\boldsymbol{\mu}}(t, \lambda) \\
& =X\left(\gamma_{t}(t, \lambda)\right) \cdot X(\boldsymbol{\mu}(t, \lambda))=\gamma_{t}(t, \lambda) \cdot \boldsymbol{\mu}(t, \lambda)=\alpha(t, \lambda), \\
\widetilde{P}_{i}(t, \lambda) & =\widetilde{\gamma}_{\lambda}(t, \lambda) \cdot \widetilde{\nu}_{i}(t, \lambda)=X\left(\gamma_{\lambda}(t, \lambda)\right) \cdot X\left(\nu_{i}(t, \lambda)\right) \\
& =\gamma_{\lambda}(t, \lambda) \cdot \nu_{i}(t, \lambda)=P_{i}(t, \lambda)(i=1, \ldots, n-1), \\
\widetilde{P}_{n}(t, \lambda) & =\widetilde{\gamma}_{\lambda}(t, \lambda) \cdot \widetilde{\boldsymbol{\mu}}(t, \lambda) \\
& =X\left(\gamma_{\lambda}(t, \lambda)\right) \cdot X(\boldsymbol{\mu}(t, \lambda))=\gamma_{\lambda}(t, \lambda) \cdot \boldsymbol{\mu}(t, \lambda)=P_{n}(t, \lambda) .
\end{aligned}
$$

Therefore, $\left(\alpha_{i j}, \beta_{i j}, \alpha, P_{1}, \ldots, P_{n}\right)$ and $\left(\widetilde{\alpha}_{i j}, \widetilde{\beta}_{i j}, \widetilde{\alpha}, \widetilde{P}_{1}, \ldots, \widetilde{P}_{n}\right)$ coincide.

Conversely, suppose that $\left(\alpha_{i j}, \beta_{i j}, \alpha, P_{1}, \ldots, P_{n}\right)$ and $\left(\widetilde{\alpha}_{i j}, \widetilde{\beta}_{i j}, \widetilde{\alpha}, \widetilde{P}_{1}, \ldots, \widetilde{P}_{n}\right)$ coincide. Let $\left(t_{0}, \lambda_{0}\right) \in I \times \Lambda$ be fixed. By using a congruence as one-parameter families of framed curves, we may assume $\gamma\left(t_{0}, \lambda_{0}\right)=\widetilde{\gamma}\left(t_{0}, \lambda_{0}\right)$ and $\boldsymbol{\nu}\left(t_{0}, \lambda_{0}\right)=$ $\widetilde{\boldsymbol{\nu}}\left(t_{0}, \lambda_{0}\right)$. By Lemma 2.8, we have $\gamma(t, \lambda)=\widetilde{\gamma}(t, \lambda)$ and $\boldsymbol{\nu}(t, \lambda)=\widetilde{\boldsymbol{\nu}}(t, \lambda)$ for all $(t, \lambda) \in I \times \Lambda$.

\section{Envelopes of one-parameter families of framed curves in the Euclidean space}

Let $(\gamma, \boldsymbol{\nu}): I \times \Lambda \rightarrow \mathbb{R}^{n} \times \Delta_{n-1}$ be a one-parameter family of framed curves with the curvature $\left(\alpha_{i j}, \beta_{i j}, \alpha, P_{1}, \ldots, P_{n}\right)$ and let $e: U \rightarrow I \times \Lambda, e(u)=(t(u), \lambda(u))$ be a smooth curve, where $U$ is an interval of $\mathbb{R}$. We denote $E_{\gamma}=\gamma \circ e: U \rightarrow \mathbb{R}^{n}$, $E_{\nu_{i}}=\nu_{i} \circ e: U \rightarrow S^{n-1}$ and $E_{\nu}=\nu \circ e: U \rightarrow \Delta_{n-1}$.

Definition 3.1. We call $E_{\gamma}$ an envelope (and $e$ a pre-envelope) for the oneparameter family of framed curves $(\gamma, \boldsymbol{\nu})$, when the following conditions are satisfied.

(i) The function $\lambda$ is non-constant on any non-trivial subinterval of $U$. (The Variability Condition.)

(ii) For all $u \in U$, the curve $E_{\gamma}$ is tangent at $u$ to the curve $\gamma(t, \lambda)$ at the parameter $(t(u), \lambda(u))$, meaning that the tangent vectors $E_{\gamma}^{\prime}(u)=$ $(d E / d u)(u)$ and $\boldsymbol{\mu}(e(u))$ are linearly dependent. (The Tangency Condition.)

This definition is a generalization of the definition of an envelope of a oneparameter family of Legendre curves in $[11,14]$. Note that the tangency condition is equivalent to the condition $E_{\gamma}^{\prime}(u) \cdot \nu_{i}(e(u))=E_{\gamma}^{\prime}(u) \cdot E_{\nu_{i}}(u)=0$ for all $u \in U$ and $i=1, \ldots, n-1$. 
Proposition 3.2. Let $(\gamma, \boldsymbol{\nu}): I \times \Lambda \rightarrow \mathbb{R}^{n} \times \Delta_{n-1}$ be a one-parameter family of framed curves with the curvature $\left(\alpha_{i j}, \beta_{i j}, \alpha, P_{1}, \ldots, P_{n}\right)$. Suppose that $e: U \rightarrow$ $I \times \Lambda, e(u)=(t(u), \lambda(u))$ is a pre-envelope and $E_{\gamma}: U \rightarrow \mathbb{R}^{n}$ is an envelope of $(\gamma, \boldsymbol{\nu})$. Then $\left(E_{\gamma}, E_{\nu}\right): U \rightarrow \mathbb{R}^{n} \times \Delta_{n-1}$ is a framed curve with the curvature

$$
\begin{aligned}
\alpha_{i j E_{\gamma}}(u) & =t^{\prime}(u) \alpha_{i j}(e(u))+\lambda^{\prime}(u) \beta_{i j}(e(u)), \\
\alpha_{E_{\gamma}}(u) & =t^{\prime}(u) \alpha(e(u))+\lambda^{\prime}(u) P_{n}(e(u)) .
\end{aligned}
$$

Proof. By definition, $E_{\nu_{i}}(u) \cdot E_{\nu_{j}}(u)=\nu_{i}(e(u)) \cdot \nu_{j}(e(u))=\delta_{i j}$ for all $u \in U$ and $i, j=1, \ldots, n-1$. Since $E_{\gamma}$ is an envelope, $E_{\gamma}^{\prime}(u) \cdot E_{\nu_{i}}(u)=0$ for all $u \in U$ and $i=1, \ldots, n-1$. It follows that $\left(E_{\gamma}, E_{\nu}\right): U \rightarrow \mathbb{R}^{n} \times \Delta_{n-1}$ is a framed curve. By a direct calculation, we have the curvature

$$
\begin{aligned}
\alpha_{i j E_{\gamma}}(u) & =E_{\nu_{i}}^{\prime}(u) \cdot E_{\nu_{j}}(e(u))=\left(t^{\prime}(u)\left(\nu_{i}\right)_{t}(e(u))+\lambda^{\prime}(u)\left(\nu_{i}\right)_{\lambda}(e(u))\right) \cdot \nu_{j}(e(u)) \\
& =t^{\prime}(u) \alpha_{i j}(e(u))+\lambda^{\prime}(u) \beta_{i j}(e(u)), \\
\alpha_{E_{\gamma}}(u) & =E_{\gamma}^{\prime}(u) \cdot \boldsymbol{\mu}(e(u))=\left(t^{\prime}(u) \gamma_{t}(e(u))+\lambda^{\prime}(u) \gamma_{\lambda}(e(u))\right) \cdot \boldsymbol{\mu}(e(u)) \\
& =t^{\prime}(u) \alpha(e(u))+\lambda^{\prime}(u) P_{n}(e(u)) .
\end{aligned}
$$

As a main result, we formulate a necessary and sufficient condition that a oneparameter family of framed curves has an envelope. The envelope theorem is as follows:

Theorem 3.3. Let $(\gamma, \boldsymbol{\nu}): I \times \Lambda \rightarrow \mathbb{R}^{n} \times \Delta_{n-1}$ be a one-parameter family of framed curves and let $e: U \rightarrow I \times \Lambda$ be a smooth curve satisfying the variability condition. Then the following are equivalent.

(1) $e: U \rightarrow I \times \Lambda$ is a pre-envelope of $(\gamma, \boldsymbol{\nu})$ and $E_{\gamma}$ is an envelope of $(\gamma, \boldsymbol{\nu})$.

(2) $\gamma_{\lambda}(e(u)) \cdot \nu_{i}(e(u))=0$ for all $u \in U$ and $i=1, \ldots, n-1$.

(3) $P_{i}(e(u))=0$ for all $u \in U$ and $i=1, \ldots, n-1$.

Proof. Suppose that $e$ is a pre-envelope of $(\gamma, \boldsymbol{\nu})$. By the tangency condition, there exists a smooth function $c: U \rightarrow \mathbb{R}$ such that $E_{\gamma}^{\prime}(u)=c(u) \boldsymbol{\mu}(e(u))$. By differentiate $E_{\gamma}(u)=\gamma \circ e(u)$, we have

$$
E_{\gamma}^{\prime}(u)=t^{\prime}(u) \gamma_{t}(e(u))+\lambda^{\prime}(u) \gamma_{\lambda}(e(u))
$$

It follows from $\gamma_{t}(t, \lambda)=\alpha(t, \lambda) \boldsymbol{\mu}(t, \lambda)$ that $\left(t^{\prime}(u) \alpha(e(u))-c(u)\right) \boldsymbol{\mu}(e(u))+$ $\lambda^{\prime}(u) \gamma_{\lambda}(e(u))=0$. Then we have $\lambda^{\prime}(u) \gamma_{\lambda}(e(u)) \cdot \nu_{i}(e(u))=0$. By the variability condition, we have $\gamma_{\lambda}(e(u)) \cdot \nu_{i}(e(u))=0$ for all $u \in U$ and $i=1, \ldots, n-1$.

Conversely, suppose that $\gamma_{\lambda}(e(u)) \cdot \nu_{i}(e(u))=0$ for all $u \in U$ and $i=1, \ldots, n-$ 1. Since

$$
E_{\gamma}^{\prime}(u) \cdot \nu_{i}(e(u))=\left(t^{\prime}(u) \gamma_{t}(e(u))+\lambda^{\prime}(u) \gamma_{\lambda}(e(u))\right) \cdot \nu_{i}(e(u))=0
$$

$e$ is a pre-envelope of $(\gamma, \boldsymbol{\nu})$. Therefore, (1) and (2) are equivalent.

By using the Frenet type formula of the one-parameter family of framed curves, $P_{i}(t, \lambda)=\gamma_{\lambda}(t, \lambda) \cdot \nu_{i}(t, \lambda)$ for all $(t, \lambda) \in I \times \Lambda$ and $i=1, \ldots, n-1$. Therefore, (2) and (3) are equivalent. 
We say that the singular set of $\gamma: I \times \Lambda \rightarrow \mathbb{R}^{n}$ is the subset of the domain $I \times \Lambda$ defined by

$$
\operatorname{rank}\left(\gamma_{t}(t, \lambda), \gamma_{\lambda}(t, \lambda)\right) \leq 1
$$

Here we discuss the relation between the envelope $E_{\gamma}$ of $(\gamma, \boldsymbol{\nu})$ and the singular set of $\gamma$. In order to consider the result, we need the following lemma.

Lemma 3.4. Let $\boldsymbol{a}, \boldsymbol{b}: U \rightarrow \mathbb{R}^{n}$ be smooth maps. Suppose that the set of nonzero points of smooth function $k: U \rightarrow \mathbb{R}$ is dense in $U$. If $k(u) \boldsymbol{a}(u)$ and $\boldsymbol{b}(u)$ are linearly dependent, then $\boldsymbol{a}(u)$ and $\boldsymbol{b}(u)$ are linearly dependent for all $u \in U$.

Proof. Since $\operatorname{rank}(k(u) \boldsymbol{a}(u), \boldsymbol{b}(u)) \leq 1$. By the condition and continuous property, we have $\operatorname{rank}(\boldsymbol{a}(u), \boldsymbol{b}(u)) \leq 1$ for all $u \in U$.

Proposition 3.5. Let $(\gamma, \boldsymbol{\nu}): I \times \Lambda \rightarrow \mathbb{R}^{n} \times \Delta_{n-1}$ be a one-parameter family of framed curves, and let $e: U \rightarrow I \times \Lambda$ be a smooth curve satisfying the variability condition. If the set of regular points of $\gamma$ on $e(U)$ is dense in $U$ and trace of e lies in the singular set of $\gamma$, then $e$ is a pre-envelope of $(\gamma, \boldsymbol{\nu})$ (and $E_{\gamma}$ is an envelope).

Proof. Since $e$ belong to the singular set of $\gamma$, we have $\operatorname{rank}\left(\gamma_{t}(e(u)), \gamma_{\lambda}(e(u))\right)$ $\leq 1$ for all $u \in U$. Therefore $\gamma_{t}(e(u))=\beta(e(u)) \boldsymbol{\mu}(e(u))$ and $\gamma_{\lambda}(e(u))$ are linearly dependent. By the assumption, the set of non-zero points of $\beta \circ e(u)$ is dense in $U$. It follows from Lemma 3.4 that $\boldsymbol{\mu}(e(u))$ and $\gamma_{\lambda}(e(u))$ are linearly dependent. Therefore $\gamma_{\lambda}(e(u)) \cdot \nu(e(u))=0$ for all $u \in U$. By Theorem 3.3,e is a pre-envelope of $(\gamma, \boldsymbol{\nu})$.

Proposition 3.6. Let $(\gamma, \boldsymbol{\nu}): I \times \Lambda \rightarrow \mathbb{R}^{n} \times \Delta_{n-1}$ be a one-parameter family of framed curves. Suppose that $e: U \rightarrow I \times \Lambda$ is a pre-envelope and $E_{\gamma}$ is an envelope of $(\gamma, \boldsymbol{\nu})$. Then $e: U \rightarrow I \times \Lambda$ is also a pre-envelope of $(-\gamma, \boldsymbol{\nu})$, $(\gamma,-\boldsymbol{\nu})$. Moreover, $-E_{\gamma}$ is an envelope of $(-\gamma, \boldsymbol{\nu})$ and $E_{\gamma}$ is an envelope of $(\gamma,-\boldsymbol{\nu})$.

Proof. Since $e: U \rightarrow I \times \Lambda$ is a pre-envelope, we have $\gamma_{\lambda}(e(u)) \cdot \nu_{i}(e(u))=0$ for all $u \in U$ and $i=1, \ldots, n-1$. It follows that $-\gamma_{\lambda}(e(u)) \cdot \nu_{i}(e(u))=0$ and $\gamma_{\lambda}(e(u)) \cdot\left(-\nu_{i}(e(u))\right)=0$ for all $u \in U$. Thus $e: U \rightarrow I \times \Lambda$ is also a pre-envelope of $(-\gamma, \boldsymbol{\nu}),(\gamma,-\boldsymbol{\nu}) .-E_{\gamma}$ is an envelope of $(-\gamma, \boldsymbol{\nu})$ and $E_{\gamma}$ is an envelope of $(\gamma,-\boldsymbol{\nu})$.

Let $\widetilde{I}$ and $\widetilde{\Lambda}$ be intervals of $\mathbb{R}$.

Definition 3.7. We say that a map $\Phi: \widetilde{I} \times \widetilde{\Lambda} \rightarrow I \times \Lambda$ is a one-parameter family of parameter change if $\Phi$ is a diffeomorphism of the form $\Phi(s, k)=$ $(\phi(s, k), \varphi(k))$. 
Proposition 3.8. Let $(\gamma, \boldsymbol{\nu}): I \times \Lambda \rightarrow \mathbb{R}^{n} \times \Delta_{n-1}$ be a one-parameter family of framed curves with the curvature $\left(\alpha_{i j}, \beta_{i j}, \alpha, P_{1}, \ldots, P_{n}\right)$. Suppose that $\Phi$ : $\widetilde{I} \times \widetilde{\Lambda} \rightarrow I \times \Lambda, \Phi(s, k)=(\phi(s, k), \varphi(k))$ is a one-parameter family of parameter change. Then $(\widetilde{\gamma}, \widetilde{\boldsymbol{\nu}})=(\gamma \circ \Phi, \boldsymbol{\nu} \circ \Phi): \widetilde{I} \times \widetilde{\Lambda} \rightarrow \mathbb{R}^{n} \times \Delta_{n-1}$ is also a one-parameter family of framed curves with the curvature

$$
\begin{aligned}
\widetilde{\alpha}_{i j}(s, k) & =\alpha_{i j}(\Phi(s, k)) \phi_{s}(s, k), \widetilde{\beta}_{i j}(s, k) \\
& =\alpha_{i j}(\Phi(s, k)) \phi_{k}(s, k)+\beta_{i j}(\Phi(s, k)) \varphi^{\prime}(k), \\
\widetilde{\alpha}(s, k) & =\alpha(\Phi(s, k)) \phi_{s}(s, k), \widetilde{P}_{i}(s, k)=P_{i}(\Phi(s, k)) \varphi^{\prime}(k)(i=1, \ldots, n-1), \\
\widetilde{P}_{n}(s, k) & =\alpha(\Phi(s, k)) \phi_{k}(s, k)+P_{n}(\Phi(s, k)) \varphi^{\prime}(k) .
\end{aligned}
$$

Moreover, if $e: U \rightarrow I \times \Lambda$ is a pre-envelope and $E_{\gamma}$ is an envelope, then $\Phi^{-1} \circ e: U \rightarrow \widetilde{I} \times \widetilde{\Lambda}$ is a pre-envelope and $E_{\gamma}$ is also an envelope of $(\widetilde{\gamma}, \widetilde{\boldsymbol{\nu}})$.

Proof. Since $\widetilde{\gamma}_{s}(s, k)=\gamma_{t}(\Phi(s, k)) \phi_{s}(s, k)$ and $\gamma_{t}(t, \lambda) \cdot \nu_{i}(t, \lambda)=0$, we have $\widetilde{\gamma}_{s}(s, k) \cdot \widetilde{\nu}_{i}(s, k)=0$ for all $(s, k) \in \widetilde{I} \times \widetilde{\Lambda}$ and $i=1, \ldots, n-1$. Therefore, $(\widetilde{\gamma}, \widetilde{\nu})$ is a one-parameter family of framed curves. By a direct calculation, we have the curvature

$$
\begin{aligned}
\widetilde{\alpha}_{i j}(s, k)= & \left(\widetilde{\nu}_{i}\right)_{s}(s, k) \cdot \widetilde{\nu}_{j}(s, k)=\left(\nu_{i}\right)_{t}(\Phi(s, k)) \phi_{s}(s, k) \cdot \nu_{j}(\Phi(s, k)) \\
= & \alpha_{i j}(\Phi(s, k)) \phi_{s}(s, k), \\
\widetilde{\beta}_{i j}(s, k)= & \left(\widetilde{\nu}_{i}\right)_{k}(s, k) \cdot \widetilde{\nu}_{j}(s, k)=\left(\left(\nu_{i}\right)_{t}(\Phi(s, k)) \phi_{k}(s, k)\right. \\
& \left.+\left(\nu_{i}\right)_{\lambda}(\Phi(s, k)) \varphi^{\prime}(k)\right) \cdot \nu_{j}(\Phi(s, k)) \\
= & \alpha_{i j}(\Phi(s, k)) \phi_{k}(s, k)+\beta_{i j}(\Phi(s, k)) \varphi^{\prime}(k), \\
\widetilde{\alpha}(s, k)= & \widetilde{\gamma}_{s}(s, k) \cdot \widetilde{\boldsymbol{\mu}}(s, k)=\gamma_{t}(\Phi(s, k)) \phi_{s}(s, k) \cdot \boldsymbol{\mu}(\Phi(s, k)) \\
= & \alpha(\Phi(s, k)) \phi_{s}(s, k), \\
\widetilde{P}_{i}(s, k)= & \widetilde{\gamma}_{k}(s, k) \cdot \widetilde{\nu}_{i}(s, k)=\left(\gamma_{t}(\Phi(s, k)) \phi_{k}(s, k)\right. \\
& \left.+\gamma_{\lambda}(\Phi(s, k)) \varphi^{\prime}(k)\right) \cdot \nu_{i}(\Phi(s, k)) \\
= & P_{i}(\Phi(s, k)) \varphi^{\prime}(k)(i=1, \ldots, n-1), \\
& \widetilde{\gamma}_{k}(s, k) \cdot \widetilde{\boldsymbol{\mu}}(s, k)=\left(\gamma_{t}(\Phi(s, k)) \phi_{k}(s, k)\right. \\
& \left.+\gamma_{\lambda}(\Phi(s, k)) \varphi^{\prime}(k)\right) \cdot \boldsymbol{\mu}(\Phi(s, k)) \\
= & \alpha(\Phi(s, k)) \phi_{k}(s, k)+P_{n}(\Phi(s, k)) \varphi^{\prime}(k) .
\end{aligned}
$$

By the form of the diffeomorphism $\Phi(s, k)=(\phi(s, k), \varphi(k)), \Phi^{-1}: I \times \Lambda \rightarrow \widetilde{I} \times$ $\widetilde{\Lambda}$ is given by the form $\Phi^{-1}(t, \lambda)=\left(\psi(t, \lambda), \varphi^{-1}(\lambda)\right)$, where $\psi$ is a smooth function. It follows that $\Phi^{-1} \circ e(u)=\left(\psi(t(u), \lambda(u)), \varphi^{-1}(\lambda(u))\right)$. Since $(d / d u) \varphi^{-1}$ $(\lambda(u))=\left(\varphi^{-1}\right)_{\lambda}(\lambda(u)) \lambda^{\prime}(u)$, the variability condition holds. Moreover, we have

$$
\begin{aligned}
& \widetilde{\gamma}_{k}(s, k) \cdot \widetilde{\nu}_{i}(s, k)=\left(\gamma_{t}(\Phi(s, k)) \phi_{k}(s, k)+\gamma_{\lambda}(\Phi(s, k)) \varphi^{\prime}(k)\right) \cdot \nu_{i}(\Phi(s, k)) \\
& =\varphi^{\prime}(k) \gamma_{\lambda}(\Phi(s, k)) \cdot \nu_{i}(\Phi(s, k)) .
\end{aligned}
$$

It follows that

$$
\widetilde{\gamma}_{k}\left(\Phi^{-1} \circ e(u)\right) \cdot \widetilde{\nu}_{i}\left(\Phi^{-1} \circ e(u)\right)=\varphi^{\prime}\left(\varphi^{-1}(\lambda(u))\right) \gamma_{\lambda}(e(u)) \cdot \nu_{i}(e(u))=0
$$


for all $u \in U$ and $i=1, \ldots, n-1$. By Theorem 3.3, $\Phi^{-1} \circ e$ is a pre-envelope of $(\widetilde{\gamma}, \widetilde{\nu})$. Therefore, $\widetilde{\gamma} \circ \Phi^{-1} \circ e=\gamma \circ \Phi \circ \Phi^{-1} \circ e=\gamma \circ e=E_{\gamma}$ is also an envelope of $(\widetilde{\gamma}, \widetilde{\boldsymbol{\nu}})$.

\section{Envelopes of one-parameter families of framed curves in $\mathbb{R}^{3} \times \Delta_{2}$}

In this section, we concentrate on the case of $n=3$. We use the following notations. Let $\left(\gamma, \nu_{1}, \nu_{2}\right): I \times \Lambda \rightarrow \mathbb{R}^{3} \times \Delta_{2}$ be a one-parameter family of framed curves and $\boldsymbol{\mu}(t, \lambda)=\nu_{1}(t, \lambda) \times \nu_{2}(t, \lambda)$. We have the Frenet type formula.s

$$
\begin{aligned}
\left(\begin{array}{c}
\nu_{1 t}(t, \lambda) \\
\nu_{2 t}(t, \lambda) \\
\boldsymbol{\mu}_{t}(t, \lambda)
\end{array}\right)= & \left(\begin{array}{ccc}
0 & \ell(t, \lambda) & m(t, \lambda) \\
-\ell(t, \lambda) & 0 & n(t, \lambda) \\
-m(t, \lambda) & -n(t, \lambda) & 0
\end{array}\right)\left(\begin{array}{c}
\nu_{1}(t, \lambda) \\
\nu_{2}(t, \lambda) \\
\boldsymbol{\mu}(t, \lambda)
\end{array}\right), \\
\left(\begin{array}{c}
\nu_{1 \lambda}(t, \lambda) \\
\nu_{2 \lambda}(t, \lambda) \\
\boldsymbol{\mu}_{\lambda}(t, \lambda)
\end{array}\right)= & \left(\begin{array}{ccc}
0 & L(t, \lambda) & M(t, \lambda) \\
-L(t, \lambda) & 0 & N(t, \lambda) \\
-M(t, \lambda) & -N(t, \lambda) & 0
\end{array}\right)\left(\begin{array}{c}
\nu_{1}(t, \lambda) \\
\nu_{2}(t, \lambda) \\
\boldsymbol{\mu}(t, \lambda)
\end{array}\right), \\
\gamma_{t}(t, \lambda)= & \alpha(t, \lambda) \boldsymbol{\mu}(t, \lambda), \gamma_{\lambda}(t, \lambda)=P(t, \lambda) \nu_{1}(t, \lambda) \\
& +Q(t, \lambda) \nu_{2}(t, \lambda)+R(t, \lambda) \boldsymbol{\mu}(t, \lambda) .
\end{aligned}
$$

Then we have the integrability condition

$$
\begin{aligned}
L_{t}(t, \lambda) & =M(t, \lambda) n(t, \lambda)-N(t, \lambda) m(t, \lambda)+\ell_{\lambda}(t, \lambda), \\
M_{t}(t, \lambda) & =N(t, \lambda) \ell(t, \lambda)-L(t, \lambda) n(t, \lambda)+m_{\lambda}(t, \lambda), \\
N_{t}(t, \lambda) & =L(t, \lambda) m(t, \lambda)-M(t, \lambda) \ell(t, \lambda)+n_{\lambda}(t, \lambda), \\
P_{t}(t, \lambda) & =Q(t, \lambda) \ell(t, \lambda)+R(t, \lambda) m(t, \lambda)-\alpha(t, \lambda) M(t, \lambda), \\
Q_{t}(t, \lambda) & =-P(t, \lambda) \ell(t, \lambda)+R(t, \lambda) n(t, \lambda)-\alpha(t, \lambda) N(t, \lambda), \\
R_{t}(t, \lambda) & =-P(t, \lambda) m(t, \lambda)-Q(t, \lambda) n(t, \lambda)+\alpha_{\lambda}(t, \lambda)
\end{aligned}
$$

for all $(t, \lambda) \in \mathrm{I} \times \Lambda$. We call the mapping $(\ell, m, n, \alpha, L, M, N, P, Q, R)$ with the integrability condition (4.1) the curvature of the one-parameter family of framed curves $\left(\gamma, \nu_{1}, \nu_{2}\right)$.

By using the above notations and Theorem 3.3, we have the following Corollary.

Corollary 4.1. Let $\left(\gamma, \nu_{1}, \nu_{2}\right): I \times \Lambda \rightarrow \mathbb{R}^{3} \times \Delta_{2}$ be a one-parameter family of framed curves with the curvature $(\ell, m, n, \alpha, L, M, N, P, Q, R)$ and let $e: U \rightarrow$ $I \times \Lambda$ be a smooth curve satisfying the variability condition. Then the following are equivalent.

(1) $e: U \rightarrow I \times \Lambda$ is a pre-envelope and $E_{\gamma}$ is an envelope of $\left(\gamma, \nu_{1}, \nu_{2}\right)$.

(2) $\gamma_{\lambda}(e(u)) \cdot \nu_{1}(e(u))=0$ and $\gamma_{\lambda}(e(u)) \cdot \nu_{2}(e(u))=0$ for all $u \in U$.

(3) $P(e(u))=0$ and $Q(e(u))=0$ for all $u \in U$. 
We give two examples. One is an example that a one-parameter family of framed curves has an envelope and the other is that a one-parameter family of framed curves does not have an envelope.

Example 4.2. Let $\left(\gamma, \nu_{1}, \nu_{2}\right): \mathbb{R} \times \mathbb{R} \rightarrow \mathbb{R}^{3} \times \Delta_{2}$ be

$$
\begin{aligned}
\gamma(t, \lambda) & =\left(\frac{1}{2} t^{2}+\lambda, \frac{1}{3} t^{3}, \frac{1}{4} t^{4}\right), \nu_{1}(t, \lambda)=\frac{(-t, 1,0)}{1+t^{2}}, \nu_{2}(t, \lambda) \\
& =\frac{\left(-t^{2},-t^{3}, 1+t^{2}\right)}{\sqrt{\left(1+t^{2}\right)\left(1+t^{2}+t^{4}\right)}} .
\end{aligned}
$$

Then $\left(\gamma, \nu_{1}, \nu_{2}\right)$ is a one-parameter family of framed curves. Since $\gamma_{\lambda}(t, \lambda)=$ $(1,0,0)$, we have $\gamma_{\lambda}(t, \lambda) \cdot \nu_{1}(t, \lambda)=-t /\left(1+t^{2}\right)$ and $\gamma_{\lambda}(t, \lambda) \cdot \nu_{2}(t, \lambda)=$ $-t^{2} / \sqrt{\left(1+t^{2}\right)\left(1+t^{2}+t^{4}\right)}$. By Corollary 4.1,e : $\mathbb{R} \rightarrow \mathbb{R} \times \mathbb{R}, e(u)=(0, u)$ is a pre-envelope of $\left(\gamma, \nu_{1}, \nu_{2}\right)$. Hence $E(u)=\gamma \circ e(u)=(u, 0,0)$ is an envelope of $\left(\gamma, \nu_{1}, \nu_{2}\right)$. For more general cases see in Example 6.1.

Example 4.3. Let $\left(\gamma, \nu_{1}, \nu_{2}\right): \mathbb{R} \times \mathbb{R} \rightarrow \mathbb{R}^{3} \times \Delta_{2}$ be

$$
\begin{aligned}
\gamma(t, \lambda) & =\left(\frac{1}{2} t^{2}, \frac{1}{3} t^{3}+\lambda, \frac{1}{4} t^{4}\right), \nu_{1}(t, \lambda)=\frac{(-t, 1,0)}{1+t^{2}}, \nu_{2}(t, \lambda) \\
& =\frac{\left(-t^{2},-t^{3}, 1+t^{2}\right)}{\sqrt{\left(1+t^{2}\right)\left(1+t^{2}+t^{4}\right)}} .
\end{aligned}
$$

Then $\left(\gamma, \nu_{1}, \nu_{2}\right)$ is also a one-parameter family of framed curves. However, since $\gamma_{\lambda}(t, \lambda)=(0,1,0)$, we have $\gamma_{\lambda}(t, \lambda) \cdot \nu_{1}(t, \lambda)=1 /\left(1+t^{2}\right) \neq 0$ for all $(t, \lambda) \in I \times \Lambda$ and $\gamma_{\lambda}(t, \lambda) \cdot \nu_{2}(t, \lambda)=-t^{3} / \sqrt{\left(1+t^{2}\right)\left(1+t^{2}+t^{4}\right)}$. By Corollary 4.1, $\left(\gamma, \nu_{1}, \nu_{2}\right)$ does not have an envelope.

\subsection{Rotated frame and reflected frame of a one-parameter family of framed curves}

Let $\left(\gamma, \nu_{1}, \nu_{2}\right): I \times \Lambda \rightarrow \mathbb{R}^{3} \times \Delta_{2}$ be a one-parameter family of framed curves with the curvature $(\ell, m, n, \alpha, L, M, N, P, Q, R)$. For the normal plane of $\gamma(t, \lambda)$, spanned by $\nu_{1}(t, \lambda)$ and $\nu_{2}(t, \lambda)$, there are other frames by rotations and reflections. We define $\left(\bar{\nu}_{1}(t, \lambda), \bar{\nu}_{2}(t, \lambda)\right) \in \Delta_{2}$ by

$$
\left(\begin{array}{c}
\bar{\nu}_{1}(t, \lambda) \\
\bar{\nu}_{2}(t, \lambda)
\end{array}\right)=\left(\begin{array}{cc}
\cos \theta(t, \lambda) & -\sin \theta(t, \lambda) \\
\sin \theta(t, \lambda) & \cos \theta(t, \lambda)
\end{array}\right)\left(\begin{array}{l}
\nu_{1}(t, \lambda) \\
\nu_{2}(t, \lambda)
\end{array}\right),
$$

where $\theta: I \times \Lambda \rightarrow \mathbb{R}$ is a smooth function. Then $\left(\gamma, \bar{\nu}_{1}, \bar{\nu}_{2}\right): I \times \Lambda \rightarrow \mathbb{R}^{3} \times$ $\Delta_{2}$ is also a one-parameter family of framed curves and $\overline{\boldsymbol{\mu}}(t, \lambda)=\bar{\nu}_{1}(t, \lambda) \times$ $\bar{\nu}_{2}(t, \lambda)=\nu_{1}(t, \lambda) \times \nu_{2}(t, \lambda)=\boldsymbol{\mu}(t, \lambda)$. By a direct calculation, the curvature $(\bar{\ell}, \bar{m}, \bar{n}, \bar{\alpha}, \bar{L}, \bar{M}, \bar{N}, \bar{P}, \bar{Q}, \bar{R})$ of $\left(\gamma, \bar{\nu}_{1}, \bar{\nu}_{2}\right)$ is given by

$\left(\ell-\theta_{t}, m \cos \theta-n \sin \theta, m \sin \theta+n \cos \theta, \alpha, L-\theta_{\lambda}, M \cos \theta-N \sin \theta\right.$,

$M \sin \theta+N \cos \theta, P \cos \theta-Q \sin \theta, P \sin \theta+Q \cos \theta, R)$.

We call the moving frame $\left\{\bar{\nu}_{1}(t, \lambda), \bar{\nu}_{2}(t, \lambda), \boldsymbol{\mu}(t, \lambda)\right\}$ a rotated frame along $\gamma(t, \lambda)$ by $\theta(t, \lambda)$. 
On the other hand, we define $\left(\widetilde{\nu}_{1}(t, \lambda), \widetilde{\nu}_{2}(t, \lambda)\right) \in \Delta_{2}$ by

$$
\left(\begin{array}{c}
\widetilde{\nu}_{1}(t, \lambda) \\
\widetilde{\nu}_{2}(t, \lambda)
\end{array}\right)=\left(\begin{array}{cc}
1 & 0 \\
0 & -1
\end{array}\right)\left(\begin{array}{cc}
\cos \theta(t, \lambda) & -\sin \theta(t, \lambda) \\
\sin \theta(t, \lambda) & \cos \theta(t, \lambda)
\end{array}\right)\left(\begin{array}{l}
\nu_{1}(t, \lambda) \\
\nu_{2}(t, \lambda)
\end{array}\right),
$$

where $\theta: I \times \Lambda \rightarrow \mathbb{R}$ is a smooth function. Then $\left(\gamma, \widetilde{\nu}_{1}, \widetilde{\nu}_{2}\right): I \times \Lambda \rightarrow \mathbb{R}^{3} \times$ $\Delta_{2}$ is also a one-parameter family of framed curves and $\widetilde{\boldsymbol{\mu}}(t, \lambda)=\widetilde{\nu}_{1}(t, \lambda) \times$ $\widetilde{\nu}_{2}(t, \lambda)=\nu_{2}(t, \lambda) \times \nu_{1}(t, \lambda)=-\boldsymbol{\mu}(t, \lambda)$. By a direct calculation, the curvature $(\widetilde{\ell}, \widetilde{m}, \widetilde{n}, \widetilde{\alpha}, \widetilde{L}, \widetilde{M}, \widetilde{N}, \widetilde{P}, \widetilde{Q}, \widetilde{R})$ of $\left(\gamma, \widetilde{\nu}_{1}, \widetilde{\nu}_{2}\right)$ is given by

$\left(-\ell+\theta_{t},-m \cos \theta+n \sin \theta, m \sin \theta+n \cos \theta,-\alpha,-L+\theta_{\lambda},-M \cos \theta+N \sin \theta\right.$,

$M \sin \theta+N \cos \theta,-P \cos \theta+Q \sin \theta, P \sin \theta+Q \cos \theta,-R)$.

We call the moving frame $\left\{\widetilde{\nu}_{1}(t, \lambda), \widetilde{\nu}_{2}(t, \lambda),-\boldsymbol{\mu}(t, \lambda)\right\}$ a reflected frame along $\gamma(t, \lambda)$ by $\theta(t, \lambda)$. By using Corollary 4.1 , we have the following result.

Proposition 4.4. Under the above notations, if e $U \rightarrow I \times \Lambda$ is a pre-envelope of $\left(\gamma, \nu_{1}, \nu_{2}\right)$, then $e: U \rightarrow I \times \Lambda$ is also a pre-envelope of $\left(\gamma, \bar{\nu}_{1}, \bar{\nu}_{2}\right)$ and $\left(\gamma, \widetilde{\nu}_{1}, \widetilde{\nu}_{2}\right)$.

Proof. By Corollary 4.1, we have $P(e(u))=Q(e(u))=0$ for all $u \in U$. It follows that $\bar{P}(e(u))=\bar{Q}(e(u))=0$ and $\widetilde{P}(e(u))=\widetilde{Q}(e(u))=0$ for all $u \in U$. Therefore, we have the result.

It follows that the envelope is independent of rotated frames and reflected frames of the framed curves.

\subsection{Parallel curves of one-parameter families of framed curves}

The parallel curve of a framed curve is defined in [9]. We define a parallel curve of a one-parameter family of framed curves.

Definition 4.5. Let $\left(\gamma, \nu_{1}, \nu_{2}\right): I \times \Lambda \rightarrow \mathbb{R}^{3} \times \Delta_{2}$ be a one-parameter family of framed curves and $\theta: I \times \Lambda \rightarrow \mathbb{R}$ be a smooth function which satisfy $\theta_{t}(t, \lambda)=\ell(t, \lambda)$ and $\theta_{\lambda}(t, \lambda)=L(t, \lambda)$ for all $(t, \lambda) \in I \times \Lambda$. Then we define a parallel curve $\gamma_{(a, b)}: I \times \Lambda \rightarrow \mathbb{R}^{3}$ of the one-parameter family of framed curves $\left(\gamma, \nu_{1}, \nu_{2}\right)$ by

$$
\begin{aligned}
\gamma_{(a, b)}(t, \lambda)= & \gamma(t, \lambda)+(a \cos \theta(t, \lambda)+b \sin \theta(t, \lambda)) \nu_{1}(t, \lambda) \\
& +(-a \sin \theta(t, \lambda)+b \cos \theta(t, \lambda)) \nu_{2}(t, \lambda)
\end{aligned}
$$

where $a, b \in \mathbb{R}$.

Remark 4.6. Since $\theta_{t}(t, \lambda)=\ell(t, \lambda)$ and $\theta_{\lambda}(t, \lambda)=L(t, \lambda), \ell_{\lambda}(t, \lambda)=L_{t}(t, \lambda)$ holds for all $(t, \lambda) \in I \times \Lambda$. By the integrability condition $(4.1), \ell_{\lambda}(t, \lambda)=$ $L_{t}(t, \lambda)$ is equivalent to $M(t, \lambda) n(t, \lambda)-N(t, \lambda) m(t, \lambda)=0$.

Proposition 4.7. Let $\left(\gamma, \nu_{1}, \nu_{2}\right): I \times \Lambda \rightarrow \mathbb{R}^{3} \times \Delta_{2}$ be a one-parameter family of framed curves with the curvature $(\ell, m, n, \alpha, L, M, N, P, Q, R)$ and $\theta: I \times \Lambda \rightarrow$ $\mathbb{R}$ be a smooth function which satisfy $\theta_{t}(t, \lambda)=\ell(t, \lambda)$ and $\theta_{\lambda}(t, \lambda)=L(t, \lambda)$ for 
all $(t, \lambda) \in I \times \Lambda$. Then $\left(\gamma_{(a, b)}, \nu_{1}, \nu_{2}\right): I \times \Lambda \rightarrow \mathbb{R}^{3} \times \Delta_{2}$ is also a one-parameter family of framed curves with the curvature $(\ell, m, n, \alpha+m(a \cos \theta+b \sin \theta)+$ $n(a \sin \theta-b \cos \theta), L, M, N, P, Q, R+M(a \cos \theta+b \sin \theta)+N(-a \sin \theta+b \cos \theta))$. Moreover, if $e: U \rightarrow I \times \Lambda$ is a pre-envelope of $\left(\gamma, \nu_{1}, \nu_{2}\right)$, then $e: U \rightarrow I \times \Lambda$ is also a pre-envelope of $\left(\gamma_{(a, b)}, \nu_{1}, \nu_{2}\right)$. We have $E_{\gamma_{(a, b)}}(u)=\left(E_{\gamma}\right)_{(a, b)}(u)$ for all $u \in U$, where $E_{\gamma_{(a, b)}}=\gamma_{(a, b)} \circ e$.

Proof. Since

$$
\begin{aligned}
\left(\gamma_{(a, b)}\right)_{t}(t, \lambda)= & \left(\alpha \boldsymbol{\mu}+\left(-a \theta_{t} \sin \theta+b \theta_{t} \cos \theta\right) \nu_{1}\right. \\
& +(a \cos \theta+b \sin \theta)\left(\ell \nu_{2}+m \boldsymbol{\mu}\right) \\
& +\left(-a \theta_{t} \cos \theta-b \theta_{t} \sin \theta\right) \nu_{2} \\
& \left.+(-a \sin \theta+b \cos \theta)\left(-\ell \nu_{1}+n \boldsymbol{\mu}\right)\right)(t, \lambda) \\
= & ((\alpha+m(a \cos \theta+b \sin \theta)+n(-a \sin \theta+b \cos \theta)) \boldsymbol{\mu})(t, \lambda), \\
\left(\gamma_{(a, b)}\right)_{\lambda}(t, \lambda)= & \left(P \nu_{1}+Q \nu_{2}+R \boldsymbol{\mu}+\left(-a \theta_{\lambda} \sin \theta\right.\right. \\
& \left.+b \theta_{\lambda} \cos \theta\right) \nu_{1}+(a \cos \theta+b \sin \theta)\left(L \nu_{2}+M \boldsymbol{\mu}\right) \\
& +\left(-a \theta_{\lambda} \cos \theta-b \theta_{\lambda} \sin \theta\right) \nu_{2} \\
& \left.+(-a \sin \theta+b \cos \theta)\left(-L \nu_{1}+N \boldsymbol{\mu}\right)\right)(t, \lambda) \\
= & \left(P \nu_{1}+Q \nu_{2}+(R+M(a \cos \theta+b \sin \theta)\right. \\
& +N(-a \sin \theta+b \cos \theta)) \boldsymbol{\mu})(t, \lambda),
\end{aligned}
$$

we have $\left(\gamma_{(a, b)}\right)_{t}(t, \lambda) \cdot \nu_{1}(t, \lambda)=0$ and $\left(\gamma_{(a, b)}\right)_{t}(t, \lambda) \cdot \nu_{2}(t, \lambda)=0$ for all $(t, \lambda) \in$ $I \times \Lambda$. Hence $\left(\gamma_{(a, b)}, \nu_{1}, \nu_{2}\right)$ is a one-parameter family of framed curves. It follows that $\gamma(t, \lambda)$ and $\gamma_{(a, b)}(t, \lambda)$ have the same moving frame $\left\{\nu_{1}(t, \lambda), \nu_{2}(t, \lambda)\right.$, $\boldsymbol{\mu}(t, \lambda)\}$. By a direct calculation, we have the curvature $(\ell, m, n, \alpha+m(a \cos \theta+$ $b \sin \theta)+n(-a \sin \theta+b \cos \theta), L, M, N, P, Q, R+M(a \cos \theta+b \sin \theta)+N(-a \sin \theta+$ $b \cos \theta))$ of $\left(\gamma_{a, b}, \nu_{1}, \nu_{2}\right)$.

If $e: U \rightarrow I \times \Lambda$ is a pre-envelope of $\left(\gamma, \nu_{1}, \nu_{2}\right)$, then $P(e(u))=0$ and $Q(e(u))=$ 0 for all $u \in U$. It follows that $\left(\gamma_{(a, b)}\right)_{\lambda}(e(u)) \cdot \nu_{1}(e(u))=0$ and $\left(\gamma_{(a, b)}\right)_{\lambda}(e(u))$. $\nu_{2}(e(u))=0$ for all $u \in U$. Thus $e: U \rightarrow I \times \Lambda$ is also a pre-envelope of $\left(\gamma_{(a, b)}, \nu_{1}, \nu_{2}\right)$ by Corollary 4.1. Moreover, we have

$$
\begin{aligned}
E_{\gamma_{(a, b)}}(u)= & \gamma_{(a, b)} \circ e(u) \\
= & \gamma(e(u))+(a \cos \theta(e(u))+b \sin \theta(e(u))) \nu_{1}(e(u)) \\
& +(-a \sin \theta(e(u))+b \cos \theta(e(u))) \nu_{2}(e(u)) \\
= & E_{\gamma}(u)+(a \cos \theta(e(u))+b \sin \theta(e(u))) E_{\nu_{1}}(u) \\
& +(-a \sin \theta(e(u))+b \cos \theta(e(u))) E_{\nu_{2}}(u) \\
= & \left(E_{\gamma}\right)_{(a, b)}(u)
\end{aligned}
$$

for all $u \in U$. 


\section{Relations among envelopes of one-parameter families of framed curves, Legendre curves and spherical Legendre curves}

First, we give relations between one-parameter families of framed curves and one-parameter families of Legendre curves in the unit tangent bundle over the Euclidean plane. We review one-parameter families of Legendre curves. For more details see [14].

Let $(\gamma, \nu): I \times \Lambda \rightarrow \mathbb{R}^{2} \times S^{1}$ be a smooth mapping. We say that $(\gamma, \nu)$ is a oneparameter family of Legendre curves if $\gamma_{t}(t, \lambda) \cdot \nu(t, \lambda)=0$ for all $(t, \lambda) \in I \times \Lambda$. We denote $\boldsymbol{J}(a)=\left(-a_{2}, a_{1}\right)$ the anticlockwise rotation by $\pi / 2$ of a vector $\boldsymbol{a}=\left(a_{1}, a_{2}\right)$. We define $\boldsymbol{\mu}(t, \lambda)=\boldsymbol{J}(\nu(t, \lambda))$. Since $\{\nu(t, \lambda), \boldsymbol{\mu}(t, \lambda)\}$ is a moving frame along $\gamma(t, \lambda)$ on $\mathbb{R}^{2}$, we have the Frenet type formula.

$$
\begin{aligned}
\left(\begin{array}{c}
\nu_{t}(t, \lambda) \\
\boldsymbol{\mu}_{t}(t, \lambda)
\end{array}\right) & =\left(\begin{array}{cc}
0 & \ell(t, \lambda) \\
-\ell(t, \lambda) & 0
\end{array}\right)\left(\begin{array}{c}
\nu(t, \lambda) \\
\boldsymbol{\mu}(t, \lambda)
\end{array}\right), \\
\left(\begin{array}{c}
\nu_{\lambda}(t, \lambda) \\
\boldsymbol{\mu}_{\lambda}(t, \lambda)
\end{array}\right) & =\left(\begin{array}{cc}
0 & L(t, \lambda) \\
-L(t, \lambda) & 0
\end{array}\right)\left(\begin{array}{c}
\nu(t, \lambda) \\
\boldsymbol{\mu}(t, \lambda)
\end{array}\right), \\
\gamma_{t}(t, \lambda) & =\alpha(t, \lambda) \boldsymbol{\mu}(t, \lambda), \gamma_{\lambda}(t, \lambda)=P(t, \lambda) \nu(t, \lambda)+Q(t, \lambda) \boldsymbol{\mu}(t, \lambda) .
\end{aligned}
$$

By the integrability condition, we have

$$
\begin{gathered}
\ell_{\lambda}(t, \lambda)=L_{t}(t, \lambda), P_{t}(t, \lambda)=\ell(t, \lambda) Q(t, \lambda)-\alpha(t, \lambda) L(t, \lambda), \alpha_{\lambda}(t, \lambda) \\
=Q_{t}(t, \lambda)+\ell(t, \lambda) P(t, \lambda)
\end{gathered}
$$

for all $(t, \lambda) \in I \times \Lambda$. We call the mapping $(\ell, \alpha, L, P, Q)$ with the integrability condition the curvature of the one-parameter family of Legendre curves $(\gamma, \nu)$.

We consider two projections to the planes. Let $\left(\gamma, \nu_{1}, \nu_{2}\right): I \times \Lambda \rightarrow \mathbb{R}^{3} \times \Delta_{2}$ be a one-parameter family of framed curves with the curvature $(\ell, m, n, \alpha, L, M, N$, $P, Q, R)$. For a fix point $\left(t_{0}, \lambda_{0}\right) \in I \times \Lambda$, we consider two projections from $\mathbb{R}^{3}$ to the $\nu_{1}\left(t_{0}, \lambda_{0}\right)$ and $\nu_{2}\left(t_{0}, \lambda_{0}\right)$ directions, respectively. We consider the projection of $\gamma$ to the $\nu_{1}\left(t_{0}, \lambda_{0}\right)$ direction. We denote

$$
\gamma_{\nu_{1}}: I \times \Lambda \rightarrow \mathbb{R}^{2},(t, \lambda) \mapsto\left(\gamma(t, \lambda) \cdot \nu_{2}\left(t_{0}, \lambda_{0}\right), \gamma(t, \lambda) \cdot \boldsymbol{\mu}\left(t_{0}, \lambda_{0}\right)\right)
$$

Then $\left(\gamma_{\nu_{1}}\right)_{t}(t, \lambda)=\alpha(t, \lambda)\left(\boldsymbol{\mu}(t, \lambda) \cdot \nu_{2}\left(t_{0}, \lambda_{0}\right), \boldsymbol{\mu}(t, \lambda) \cdot \boldsymbol{\mu}\left(t_{0}, \lambda_{0}\right)\right)$. There exist subintervals $I_{1}$ of $I$ around $t_{0}$ and $\Lambda_{1}$ of $\Lambda$ around $\lambda_{0}$ such that $(\boldsymbol{\mu}(t, \lambda)$. $\left.\nu_{2}\left(t_{0}, \lambda_{0}\right)\right)^{2}+\left(\boldsymbol{\mu}(t, \lambda) \cdot \boldsymbol{\mu}\left(t_{0}, \lambda_{0}\right)\right)^{2} \neq 0$ for all $(t, \lambda) \in I_{1} \times \Lambda_{1}$.

Proposition 5.1. Let $\left(\gamma, \nu_{1}, \nu_{2}\right): I \times \Lambda \rightarrow \mathbb{R}^{3} \times \Delta_{2}$ be a one-parameter family of framed curves with the curvature $(\ell, m, n, \alpha, L, M, N, P, Q, R)$. Then $\left(\gamma_{\nu_{1}}, \nu_{\nu_{1}}\right)$ : $I_{1} \times \Lambda_{1} \rightarrow \mathbb{R}^{2} \times S^{1}$ is a one-parameter family of Legendre curves with the 
curvature $\left(\ell_{\nu_{1}}, \alpha_{\nu_{1}}, L_{\nu_{1}}, P_{\nu_{1}}, Q_{\nu_{1}}\right)$, where

$$
\begin{aligned}
& \nu_{\nu_{1}}(t, \lambda)=\frac{1}{\sqrt{\left(\boldsymbol{\mu} \cdot \nu_{2}\left(t_{0}, \lambda_{0}\right)\right)^{2}+\left(\boldsymbol{\mu} \cdot \boldsymbol{\mu}\left(t_{0}, \lambda_{0}\right)\right)^{2}}}\left(\boldsymbol{\mu} \cdot \boldsymbol{\mu}\left(t_{0}, \lambda_{0}\right),\right. \\
& \left.-\boldsymbol{\mu} \cdot \nu_{2}\left(t_{0}, \lambda_{0}\right)\right)(t, \lambda) \text {, } \\
& \ell_{\nu_{1}}(t, \lambda)=\frac{1}{\left(\boldsymbol{\mu} \cdot \nu_{2}\left(t_{0}, \lambda_{0}\right)\right)^{2}+\left(\boldsymbol{\mu} \cdot \boldsymbol{\mu}\left(t_{0}, \lambda_{0}\right)\right)^{2}} \\
& \left(\ell\left(\left(\nu_{1} \cdot \nu_{2}\left(t_{0}, \lambda_{0}\right)\right)\left(\boldsymbol{\mu} \cdot \boldsymbol{\mu}\left(t_{0}, \lambda_{0}\right)\right)-\left(\nu_{1} \cdot \boldsymbol{\mu}\left(t_{0}, \lambda_{0}\right)\right)\left(\boldsymbol{\mu} \cdot \nu_{2}\left(t_{0}, \lambda_{0}\right)\right)\right)\right. \\
& +n\left(\left(\nu_{2} \cdot \nu_{2}\left(t_{0}, \lambda_{0}\right)\right)\left(\boldsymbol{\mu} \cdot \boldsymbol{\mu}\left(t_{0}, \lambda_{0}\right)\right)\right. \\
& \left.\left.-\left(\nu_{2} \cdot \boldsymbol{\mu}\left(t_{0}, \lambda_{0}\right)\right)\left(\boldsymbol{\mu} \cdot \nu_{2}\left(t_{0}, \lambda_{0}\right)\right)\right)\right)(t, \lambda), \\
& \alpha_{\nu_{1}}(t, \lambda)=\alpha \sqrt{\left(\boldsymbol{\mu} \cdot \nu_{2}\left(t_{0}, \lambda_{0}\right)\right)^{2}+\left(\boldsymbol{\mu} \cdot \boldsymbol{\mu}\left(t_{0}, \lambda_{0}\right)\right)^{2}}(t, \lambda), \\
& L_{\nu_{1}}(t, \lambda)=\frac{1}{\left(\boldsymbol{\mu} \cdot \nu_{2}\left(t_{0}, \lambda_{0}\right)\right)^{2}+\left(\boldsymbol{\mu} \cdot \boldsymbol{\mu}\left(t_{0}, \lambda_{0}\right)\right)^{2}} \\
& \left(L\left(\left(\nu_{1} \cdot \nu_{2}\left(t_{0}, \lambda_{0}\right)\right)\left(\boldsymbol{\mu} \cdot \boldsymbol{\mu}\left(t_{0}, \lambda_{0}\right)\right)-\left(\nu_{1} \cdot \boldsymbol{\mu}\left(t_{0}, \lambda_{0}\right)\right)\left(\boldsymbol{\mu} \cdot \nu_{2}\left(t_{0}, \lambda_{0}\right)\right)\right)\right. \\
& +N\left(\left(\nu_{2} \cdot \nu_{2}\left(t_{0}, \lambda_{0}\right)\right)\left(\boldsymbol{\mu} \cdot \boldsymbol{\mu}\left(t_{0}, \lambda_{0}\right)\right)\right. \\
& \left.\left.-\left(\nu_{2} \cdot \boldsymbol{\mu}\left(t_{0}, \lambda_{0}\right)\right)\left(\boldsymbol{\mu} \cdot \nu_{2}\left(t_{0}, \lambda_{0}\right)\right)\right)\right)(t, \lambda), \\
& P_{\nu_{1}}(t, \lambda)=\frac{1}{\sqrt{\left(\boldsymbol{\mu} \cdot \nu_{2}\left(t_{0}, \lambda_{0}\right)\right)^{2}+\left(\boldsymbol{\mu} \cdot \boldsymbol{\mu}\left(t_{0}, \lambda_{0}\right)\right)^{2}}} \\
& \left(P\left(\left(\nu_{1} \cdot \nu_{2}\left(t_{0}, \lambda_{0}\right)\right)\left(\boldsymbol{\mu} \cdot \boldsymbol{\mu}\left(t_{0}, \lambda_{0}\right)\right)-\left(\nu_{1} \cdot \boldsymbol{\mu}\left(t_{0}, \lambda_{0}\right)\right)\left(\boldsymbol{\mu} \cdot \nu_{2}\left(t_{0}, \lambda_{0}\right)\right)\right)\right. \\
& +Q\left(\left(\nu_{2} \cdot \nu_{2}\left(t_{0}, \lambda_{0}\right)\right)\left(\boldsymbol{\mu} \cdot \boldsymbol{\mu}\left(t_{0}, \lambda_{0}\right)\right)\right. \\
& \left.\left.-\left(\nu_{2} \cdot \boldsymbol{\mu}\left(t_{0}, \lambda_{0}\right)\right)\left(\boldsymbol{\mu} \cdot \nu_{2}\left(t_{0}, \lambda_{0}\right)\right)\right)\right)(t, \lambda), \\
& Q_{\nu_{1}}(t, \lambda)=\frac{1}{\sqrt{\left(\boldsymbol{\mu} \cdot \nu_{2}\left(t_{0}, \lambda_{0}\right)\right)^{2}+\left(\boldsymbol{\mu} \cdot \boldsymbol{\mu}\left(t_{0}, \lambda_{0}\right)\right)^{2}}} \\
& \left(P \left(\left(\nu_{1} \cdot \nu_{2}\left(t_{0}, \lambda_{0}\right)\right)\left(\boldsymbol{\mu} \cdot \nu_{2}\left(t_{0}, \lambda_{0}\right)\right)\right.\right. \\
& \left.+\left(\nu_{1} \cdot \boldsymbol{\mu}\left(t_{0}, \lambda_{0}\right)\right)\left(\boldsymbol{\mu} \cdot \boldsymbol{\mu}\left(t_{0}, \lambda_{0}\right)\right)\right) \\
& +Q\left(\left(\nu_{2} \cdot \nu_{2}\left(t_{0}, \lambda_{0}\right)\right)\left(\boldsymbol{\mu} \cdot \nu_{2}\left(t_{0}, \lambda_{0}\right)\right)+\left(\nu_{2} \cdot \boldsymbol{\mu}\left(t_{0}, \lambda_{0}\right)\right)\left(\boldsymbol{\mu} \cdot \boldsymbol{\mu}\left(t_{0}, \lambda_{0}\right)\right)\right) \\
& \left.+R\left(\left(\boldsymbol{\mu} \cdot \nu_{2}\left(t_{0}, \lambda_{0}\right)\right)^{2}+\left(\boldsymbol{\mu} \cdot \boldsymbol{\mu}\left(t_{0}, \lambda_{0}\right)\right)^{2}\right)\right)(t, \lambda) \text {. }
\end{aligned}
$$

Proof. We define a smooth map $\nu_{\nu_{1}}: I_{1} \times \Lambda_{1} \rightarrow S^{1}$ by

$$
\begin{aligned}
\nu_{\nu_{1}}(t, \lambda)= & \frac{1}{\sqrt{\left(\boldsymbol{\mu} \cdot \nu_{2}\left(t_{0}, \lambda_{0}\right)\right)^{2}+\left(\boldsymbol{\mu} \cdot \boldsymbol{\mu}\left(t_{0}, \lambda_{0}\right)\right)^{2}}}\left(\boldsymbol{\mu} \cdot \boldsymbol{\mu}\left(t_{0}, \lambda_{0}\right),\right. \\
& \left.-\boldsymbol{\mu} \cdot \nu_{2}\left(t_{0}, \lambda_{0}\right)\right)(t, \lambda) .
\end{aligned}
$$

Then $\left(\gamma_{\nu_{1}}, \nu_{\nu_{1}}\right): I_{1} \times \Lambda_{1} \rightarrow \mathbb{R}^{2} \times S^{1}$ is a one-parameter family of Legendre curves. By definition, $\boldsymbol{\mu}_{\nu_{1}}: I_{1} \times \Lambda_{1} \rightarrow S^{1}$ is given by

$$
\begin{aligned}
& \boldsymbol{\mu}_{\nu_{1}}(t, \lambda) \\
& =J\left(\nu_{\nu_{1}}(t, \lambda)\right)=\frac{1}{\sqrt{\left(\boldsymbol{\mu} \cdot \nu_{2}\left(t_{0}, \lambda_{0}\right)\right)^{2}+\left(\boldsymbol{\mu} \cdot \boldsymbol{\mu}\left(t_{0}, \lambda_{0}\right)\right)^{2}}} \\
& \quad\left(\boldsymbol{\mu} \cdot \nu_{2}\left(t_{0}, \lambda_{0}\right), \boldsymbol{\mu} \cdot \boldsymbol{\mu}\left(t_{0}, \lambda_{0}\right)\right)(t, \lambda) .
\end{aligned}
$$


By a direct calculation, we have the curvature $\left(\ell_{\nu_{1}}, \alpha_{\nu_{1}}, L_{\nu_{1}}, P_{\nu_{1}}, Q_{\nu_{1}}\right)$ of $\left(\gamma_{\nu_{1}}, \nu_{\nu_{1}}\right)$.

Proposition 5.2. Under the same notations in Proposition 5.1, suppose that $e: U \rightarrow I_{1} \times \Lambda_{1}$ is a pre-envelope of $\left(\gamma, \nu_{1}, \nu_{2}\right)$. Then $e: U \rightarrow I_{1} \times \Lambda_{1}$ is also a pre-envelope of $\left(\gamma_{\nu_{1}}, \nu_{\nu_{1}}\right): I_{1} \times \Lambda_{1} \rightarrow \mathbb{R}^{2} \times S^{1}$. Moreover, we have $E_{\gamma_{\nu_{1}}}(u)=\left(E_{\gamma}\right)_{\nu_{1}}(u)$ for all $u \in U$, where $E_{\gamma_{\nu_{1}}}(u)=\gamma_{\nu_{1}} \circ e(u)$.

Proof. Since $\left(\gamma, \nu_{1}, \nu_{2}\right): I \times \Lambda \rightarrow \mathbb{R}^{3} \times \Delta_{2}$ is a one-parameter family of framed curves and $e: U \rightarrow I_{1} \times \Lambda_{1}$ is a pre-envelope of $\left(\gamma, \nu_{1}, \nu_{2}\right)$, we have $P(e(u))=Q(e(u))=0$ for all $u \in U$ by Corollary 4.1. By Proposition 5.1, we have $P_{\nu_{1}}(e(u))=0$ for all $u \in U$. Therefore $e: U \rightarrow I_{1} \times \Lambda_{1}$ is a preenvelope of $\left(\gamma_{\nu_{1}}, \nu_{\nu_{1}}\right)$ (cf. [14]). Moreover, we have $E_{\gamma_{\nu_{1}}}(u)=\gamma_{\nu_{1}} \circ e(u)=$ $\left(\gamma(e(u)) \cdot \nu_{2}\left(t_{0}, \lambda_{0}\right), \gamma(e(u)) \cdot \boldsymbol{\mu}\left(t_{0}, \lambda_{0}\right)\right)=\left(E_{\gamma}(u) \cdot \nu_{2}\left(t_{0}, \lambda_{0}\right), E_{\gamma}(u) \cdot \boldsymbol{\mu}\left(t_{0}, \lambda_{0}\right)\right)=$ $\left(E_{\gamma}\right)_{\nu_{1}}(u)$ for all $u \in U$.

We also consider the projection of $\gamma$ to the $\nu_{2}\left(t_{0}, \lambda_{0}\right)$ direction. We denote $\gamma_{\nu_{2}}: I \times \Lambda \rightarrow \mathbb{R}^{2},(t, \lambda) \mapsto\left(\gamma(t, \lambda) \cdot \nu_{1}\left(t_{0}, \lambda_{0}\right), \gamma(t, \lambda) \cdot \boldsymbol{\mu}\left(t_{0}, \lambda_{0}\right)\right)$. There exist subintervals $I_{2}$ of $I$ around $t_{0}$ and $\Lambda_{2}$ of $\Lambda$ around $\lambda_{0}$ such that $(\boldsymbol{\mu}(t, \lambda)$. $\left.\nu_{1}\left(t_{0}, \lambda_{0}\right)\right)^{2}+\left(\boldsymbol{\mu}(t, \lambda) \cdot \boldsymbol{\mu}\left(t_{0}, \lambda_{0}\right)\right)^{2} \neq 0$ for all $(t, \lambda) \in I_{2} \times \Lambda_{2}$. We can prove the following similarly.

Proposition 5.3. Let $\left(\gamma, \nu_{1}, \nu_{2}\right): I \times \Lambda \rightarrow \mathbb{R}^{3} \times \Delta_{2}$ be a one-parameter family of framed curves with the curvature $(\ell, m, n, \alpha, L, M, N, P, Q, R)$. Then $\left(\gamma_{\nu_{2}}, \nu_{\nu_{2}}\right)$ : $I_{2} \times \Lambda_{2} \rightarrow \mathbb{R}^{2} \times S^{1}$ is a one-parameter family of Legendre curves with the curvature $\left(\ell_{\nu_{2}}, \alpha_{\nu_{2}}, L_{\nu_{2}}, P_{\nu_{2}}, Q_{\nu_{2}}\right)$, where

$$
\begin{aligned}
\nu_{\nu_{2}}(t, \lambda)= & \frac{1}{\sqrt{\left(\boldsymbol{\mu} \cdot \nu_{1}\left(t_{0}, \lambda_{0}\right)\right)^{2}+\left(\boldsymbol{\mu} \cdot \boldsymbol{\mu}\left(t_{0}, \lambda_{0}\right)\right)^{2}}} \\
& \times\left(\boldsymbol{\mu} \cdot \boldsymbol{\mu}\left(t_{0}, \lambda_{0}\right),-\boldsymbol{\mu} \cdot \nu_{1}\left(t_{0}, \lambda_{0}\right)\right)(t, \lambda), \\
\ell_{\nu_{2}}(t, \lambda)= & \frac{1}{\left(\boldsymbol{\mu} \cdot \nu_{1}\left(t_{0}, \lambda_{0}\right)\right)^{2}+\left(\boldsymbol{\mu} \cdot \boldsymbol{\mu}\left(t_{0}, \lambda_{0}\right)\right)^{2}} \\
& \left(\ell\left(\left(\nu_{1} \cdot \nu_{1}\left(t_{0}, \lambda_{0}\right)\right)\left(\boldsymbol{\mu} \cdot \boldsymbol{\mu}\left(t_{0}, \lambda_{0}\right)\right)-\left(\nu_{1} \cdot \boldsymbol{\mu}\left(t_{0}, \lambda_{0}\right)\right)\left(\boldsymbol{\mu} \cdot \nu_{1}\left(t_{0}, \lambda_{0}\right)\right)\right)\right. \\
& \left.+n\left(\left(\nu_{2} \cdot \nu_{1}\left(t_{0}, \lambda_{0}\right)\right)\left(\boldsymbol{\mu} \cdot \boldsymbol{\mu}\left(t_{0}, \lambda_{0}\right)\right)-\left(\nu_{2} \cdot \boldsymbol{\mu}\left(t_{0}, \lambda_{0}\right)\right)\left(\boldsymbol{\mu} \cdot \nu_{1}\left(t_{0}, \lambda_{0}\right)\right)\right)\right) \\
& \times(t, \lambda), \\
\alpha_{\nu_{2}}(t, \lambda)= & \alpha \sqrt{\left(\boldsymbol{\mu} \cdot \nu_{1}\left(t_{0}, \lambda_{0}\right)\right)^{2}+\left(\boldsymbol{\mu} \cdot \boldsymbol{\mu}\left(t_{0}, \lambda_{0}\right)\right)^{2}}(t, \lambda), \\
L_{\nu_{2}}(t, \lambda)= & \frac{1}{\left(\boldsymbol{\mu} \cdot \nu_{1}\left(t_{0}, \lambda_{0}\right)\right)^{2}+\left(\boldsymbol{\mu} \cdot \boldsymbol{\mu}\left(t_{0}, \lambda_{0}\right)\right)^{2}} \\
& \left(L\left(\left(\nu_{1} \cdot \nu_{1}\left(t_{0}, \lambda_{0}\right)\right)\left(\boldsymbol{\mu} \cdot \boldsymbol{\mu}\left(t_{0}, \lambda_{0}\right)\right)-\left(\nu_{2} \cdot \boldsymbol{\mu}\left(t_{0}, \lambda_{0}\right)\right)\left(\boldsymbol{\mu} \cdot \nu_{1}\left(t_{0}, \lambda_{0}\right)\right)\right)\right. \\
& \left.+N\left(\left(\nu_{2} \cdot \nu_{1}\left(t_{0}, \lambda_{0}\right)\right)\left(\boldsymbol{\mu} \cdot \boldsymbol{\mu}\left(t_{0}, \lambda_{0}\right)\right)-\left(\nu_{2} \cdot \boldsymbol{\mu}\left(t_{0}, \lambda_{0}\right)\right)\left(\boldsymbol{\mu} \cdot \nu_{1}\left(t_{0}, \lambda_{0}\right)\right)\right)\right) \\
& \times(t, \lambda),
\end{aligned}
$$




$$
\begin{aligned}
P_{\nu_{2}}(t, \lambda)= & \frac{1}{\sqrt{\left(\boldsymbol{\mu} \cdot \nu_{1}\left(t_{0}, \lambda_{0}\right)\right)^{2}+\left(\boldsymbol{\mu} \cdot \boldsymbol{\mu}\left(t_{0}, \lambda_{0}\right)\right)^{2}}} \\
& \left(P\left(\left(\nu_{1} \cdot \nu_{1}\left(t_{0}, \lambda_{0}\right)\right)\left(\boldsymbol{\mu} \cdot \boldsymbol{\mu}\left(t_{0}, \lambda_{0}\right)\right)-\left(\nu_{1} \cdot \boldsymbol{\mu}\left(t_{0}, \lambda_{0}\right)\right)\left(\boldsymbol{\mu} \cdot \nu_{1}\left(t_{0}, \lambda_{0}\right)\right)\right)\right. \\
& \left.+Q\left(\left(\nu_{2} \cdot \nu_{1}\left(t_{0}, \lambda_{0}\right)\right)\left(\boldsymbol{\mu} \cdot \boldsymbol{\mu}\left(t_{0}, \lambda_{0}\right)\right)-\left(\nu_{2} \cdot \boldsymbol{\mu}\left(t_{0}, \lambda_{0}\right)\right)\left(\boldsymbol{\mu} \cdot \nu_{1}\left(t_{0}, \lambda_{0}\right)\right)\right)\right) \\
& \times(t, \lambda), \\
Q_{\nu_{2}}(t, \lambda)= & \frac{1}{\sqrt{\left(\boldsymbol{\mu} \cdot \nu_{1}\left(t_{0}, \lambda_{0}\right)\right)^{2}+\left(\boldsymbol{\mu} \cdot \boldsymbol{\mu}\left(t_{0}, \lambda_{0}\right)\right)^{2}}} \\
& \left(P\left(\left(\nu_{1} \cdot \nu_{1}\left(t_{0}, \lambda_{0}\right)\right)\left(\boldsymbol{\mu} \cdot \nu_{1}\left(t_{0}, \lambda_{0}\right)\right)+\left(\nu_{1} \cdot \boldsymbol{\mu}\left(t_{0}, \lambda_{0}\right)\right)\left(\boldsymbol{\mu} \cdot \boldsymbol{\mu}\left(t_{0}, \lambda_{0}\right)\right)\right)\right. \\
& +Q\left(\left(\nu_{2} \cdot \nu_{1}\left(t_{0}, \lambda_{0}\right)\right)\left(\boldsymbol{\mu} \cdot \nu_{1}\left(t_{0}, \lambda_{0}\right)\right)+\left(\nu_{2} \cdot \boldsymbol{\mu}\left(t_{0}, \lambda_{0}\right)\right)\left(\boldsymbol{\mu} \cdot \boldsymbol{\mu}\left(t_{0}, \lambda_{0}\right)\right)\right) \\
& \left.+R\left(\left(\boldsymbol{\mu} \cdot \nu_{1}\left(t_{0}, \lambda_{0}\right)\right)^{2}+\left(\boldsymbol{\mu} \cdot \boldsymbol{\mu}\left(t_{0}, \lambda_{0}\right)\right)^{2}\right)\right)(t, \lambda) .
\end{aligned}
$$

Proposition 5.4. Under the same notations in Proposition 5.3, suppose that $e: U \rightarrow I_{2} \times \Lambda_{2}$ is a pre-envelope of $\left(\gamma, \nu_{1}, \nu_{2}\right)$. Then $e: U \rightarrow I_{2} \times \Lambda_{2}$ is also a pre-envelope of $\left(\gamma_{\nu_{2}}, \nu_{\nu_{2}}\right): I_{2} \times \Lambda_{2} \rightarrow \mathbb{R}^{2} \times S^{1}$. Moreover, we have $E_{\gamma_{\nu_{2}}}(u)=\left(E_{\gamma}\right)_{\nu_{2}}(u)$ for all $u \in U$, where $E_{\gamma_{\nu_{2}}}(u)=\gamma_{\nu_{2}} \circ e(u)$.

Second, we give a relations between one-parameter families of framed curves and one-parameter families of spherical Legendre curves in the unit spherical bundle over the unit sphere. We review the one-parameter families of spherical Legendre curves. For more details see [11].

Let $(\gamma, \nu): I \times \Lambda \rightarrow \Delta_{2} \subset S^{2} \times S^{2}$ be a smooth mapping. We say that $(\gamma, \nu)$ is a one-parameter family of spherical Legendre curves if $\gamma_{t}(t, \lambda) \cdot \nu(t, \lambda)=0$ for all $(t, \lambda) \in I \times \Lambda$. We define $\boldsymbol{\mu}(t, \lambda)=\gamma(t, \lambda) \times \nu(t, \lambda)$. Then $\{\gamma(t, \lambda), \nu(t, \lambda), \boldsymbol{\mu}(t, \lambda)\}$ is a moving frame along $\gamma(t, \lambda)$ on $S^{2}$. We have the Frenet type formula.

$$
\begin{aligned}
\left(\begin{array}{c}
\gamma_{t}(t, \lambda) \\
\nu_{t}(t, \lambda) \\
\boldsymbol{\mu}_{t}(t, \lambda)
\end{array}\right) & =\left(\begin{array}{ccc}
0 & 0 & m(t, \lambda) \\
0 & 0 & n(t, \lambda) \\
-m(t, \lambda) & -n(t, \lambda) & 0
\end{array}\right)\left(\begin{array}{c}
\gamma(t, \lambda) \\
\nu(t, \lambda) \\
\boldsymbol{\mu}(t, \lambda)
\end{array}\right), \\
\left(\begin{array}{c}
\gamma_{\lambda}(t, \lambda) \\
\nu_{\lambda}(t, \lambda) \\
\boldsymbol{\mu}_{\lambda}(t, \lambda)
\end{array}\right) & =\left(\begin{array}{ccc}
0 & L(t, \lambda) & M(t, \lambda) \\
-L(t, \lambda) & 0 & N(t, \lambda) \\
-M(t, \lambda) & -N(t, \lambda) & 0
\end{array}\right)\left(\begin{array}{c}
\gamma(t, \lambda) \\
\nu(t, \lambda) \\
\boldsymbol{\mu}(t, \lambda)
\end{array}\right) .
\end{aligned}
$$

By the integrability condition, we have

$$
\begin{aligned}
& L_{t}(t, \lambda)=M(t, \lambda) n(t, \lambda)-N(t, \lambda) m(t, \lambda), \\
& m_{\lambda}(t, \lambda)=M_{t}(t, \lambda)+L(t, \lambda) n(t, \lambda), \\
& n_{\lambda}(t, \lambda)=N_{t}(t, \lambda)-L(t, \lambda) m(t, \lambda) .
\end{aligned}
$$

We call the mapping $(m, n, L, M, N)$ with the integrability condition the curvature of the one-parameter family of spherical Legendre curves $(\gamma, \nu)$. Let $(\gamma, \nu): I \times \Lambda \rightarrow \Delta_{2}$ be a one-parameter family of spherical Legendre curves with the curvature $(m, n, L, M, N)$ and let $e: U \rightarrow I \times \Lambda, e(u)=(t(u), \lambda(u))$ be a smooth curve, where $U$ is an interval of $\mathbb{R}$. We denote $E_{\gamma}=\gamma \circ e: U \rightarrow S^{2}$ and $E_{\nu}=\nu \circ e: U \rightarrow S^{2}$.

We call $E_{\gamma}$ an envelope (and $e$ a pre-envelope) for the one-parameter family of spherical Legendre curves $(\gamma, \nu)$, when the following conditions are satisfied. 
(i) The function $\lambda$ is non-constant on any non-trivial subinterval of $U$. (The Variability Condition.)

(ii) For all $u$, the curve $E_{\gamma}$ is tangent at $u$ to the curve $\gamma(t, \lambda)$ at the parameter $(t(u), \lambda(u))$, meaning that the tangent vectors $E_{\gamma}^{\prime}(u)=(d E / d u)(u)$ and $\boldsymbol{\mu}(e(u))$ are linearly dependent. (The Tangency Condition.)

In [13], the relations between framed curves and spherical Legendre curves are discussed. Here we discuss relations between one-parameter families of framed curves and one-parameter families of spherical Legendre curves, see Example 6.4.

Proposition 5.5. Let $\left(\gamma, \nu_{1}, \nu_{2}\right): I \times \Lambda \rightarrow \mathbb{R}^{3} \times \Delta_{2}$ be a one-parameter family of framed curves with the curvature $(\ell, m, n, \alpha, L, M, N, P, Q, R)$. Suppose that $\gamma(t, \lambda)$ is non-zero. We denote $\widetilde{\gamma}(t, \lambda)=\gamma(t, \lambda) /|\gamma(t, \lambda)|$ and $\widetilde{\gamma}(t, \lambda)=$ $a(t, \lambda) \nu_{1}(t, \lambda)+b(t, \lambda) \nu_{2}(t, \lambda)+c(t, \lambda) \boldsymbol{\mu}(t, \lambda)$ with $a^{2}(t, \lambda)+b^{2}(t, \lambda)+c^{2}(t, \lambda)=1$. If $a^{2}(t, \lambda)+b^{2}(t, \lambda) \neq 0$ for all $(t, \lambda) \in I \times \Lambda$ and $\widetilde{\nu}(t, \lambda)=(\widetilde{\gamma} \times \boldsymbol{\mu} /|\widetilde{\gamma} \times \boldsymbol{\mu}|)(t, \lambda)$, then $(\widetilde{\gamma}, \widetilde{\nu}): I \times \Lambda \rightarrow \Delta_{2}$ is a one-parameter family of spherical Legendre curves with the curvature

$$
\begin{aligned}
\widetilde{m}(t, \lambda) & =-\frac{a m+b n+c_{t}}{\sqrt{a^{2}+b^{2}}}(t, \lambda), \\
\widetilde{n}(t, \lambda) & =\frac{\left(a^{2}+b^{2}\right)(a n-b m+c \ell)+\left(a b_{t}-a_{t} b\right) c}{\sqrt{a^{2}+b^{2}}}(t, \lambda), \\
\widetilde{L}(t, \lambda) & =\frac{-a\left(b_{\lambda}+a L-c N\right)+b\left(a_{\lambda}-b L-c M\right)}{\sqrt{a^{2}+b^{2}}}(t, \lambda), \\
\widetilde{M}(t, \lambda) & =-\frac{a M+b N+c_{\lambda}}{\sqrt{a^{2}+b^{2}}}(t, \lambda), \\
\widetilde{N}(t, \lambda) & =\frac{\left(a^{2}+b^{2}\right)(a N-b M+c L)+\left(a b_{\lambda}-a_{\lambda} b\right) c}{\sqrt{a^{2}+b^{2}}}(t, \lambda) .
\end{aligned}
$$

Proof. Since $\widetilde{\gamma}(t, \lambda)=a(t, \lambda) \nu_{1}(t, \lambda)+b(t, \lambda) \nu_{2}(t, \lambda)+c(t, \lambda) \boldsymbol{\mu}(t, \lambda)$ and $\widetilde{\nu}(t, \lambda)$ $=\left(\left(b \nu_{1}-a \nu_{2}\right) / \sqrt{a^{2}+b^{2}}\right)(t, \lambda)$, we have $\widetilde{\gamma}(t, \lambda) \cdot \widetilde{\nu}(t, \lambda)=0$. Moreover,

$$
\begin{aligned}
\widetilde{\gamma}_{t}(t, \lambda) \cdot \widetilde{\nu}(t, \lambda) & =\left(\frac{\gamma_{t}|\gamma|-\gamma|\gamma|_{t}}{|\gamma|^{2}} \cdot \widetilde{\nu}\right)(t, \lambda)=\left(\frac{\gamma_{t}}{|\gamma|} \cdot \widetilde{\nu}-\frac{|\gamma|_{t}}{|\gamma|} \widetilde{\gamma} \cdot \widetilde{\nu}\right)(t, \lambda) \\
& =\frac{\gamma_{t} \cdot\left(b \nu_{1}-a \nu_{2}\right)}{|\gamma| \sqrt{a^{2}+b^{2}}}(t, \lambda)=0 .
\end{aligned}
$$

Therefore $(\widetilde{\gamma}, \widetilde{\nu}): I \times \Lambda \rightarrow \Delta_{2}$ is a one-parameter family of spherical Legendre curves. By a direct calculation, we have

$$
\widetilde{\boldsymbol{\mu}}(t, \lambda)=\widetilde{\gamma}(t, \lambda) \times \widetilde{\nu}(t, \lambda)=\frac{1}{\sqrt{a^{2}+b^{2}}}\left(a c \nu_{1}+b c \nu_{2}-\left(a^{2}+b^{2}\right) \boldsymbol{\mu}\right)(t, \lambda)
$$


By using the Frenet type formula of a one-parameter family of spherical Legendre curves, we also have

$$
\begin{aligned}
\widetilde{\gamma}_{t}(t, \lambda)= & \left(\left(a_{t}-b \ell-c m\right) \nu_{1}+\left(b_{t}+a \ell-c n\right) \nu_{2}+\left(c_{t}+a m+b n\right) \boldsymbol{\mu}\right)(t, \lambda), \\
\widetilde{\gamma}_{\lambda}(t, \lambda)= & \left(\left(a_{\lambda}-b L-c M\right) \nu_{1}+\left(b_{\lambda}+a L-c N\right) \nu_{2}+\left(c_{\lambda}+a M+b N\right) \boldsymbol{\mu}\right)(t, \lambda), \\
\widetilde{\nu}_{t}(t, \lambda)= & \frac{1}{\left(a^{2}+b^{2}\right)^{\frac{3}{2}}}\left(\left(b_{t} a^{2}+a\left(a^{2}+b^{2}\right) \ell-a_{t} a b\right) \nu_{1}\right. \\
& \left.+\left(-a_{t} b^{2}+b\left(a^{2}+b^{2}\right) \ell+b_{t} a b\right) \nu_{2}+\left(a^{2}+b^{2}\right)(-a n+b m) \boldsymbol{\mu}\right)(t, \lambda), \\
\widetilde{\nu}_{\lambda}(t, \lambda)= & \frac{1}{\left(a^{2}+b^{2}\right)^{\frac{3}{2}}}\left(\left(b_{\lambda} a^{2}+a\left(a^{2}+b^{2}\right) L-a_{\lambda} a b\right) \nu_{1}\right. \\
& \left.+\left(-a_{\lambda} b^{2}+b\left(a^{2}+b^{2}\right) L+b_{\lambda} a b\right) \nu_{2}+\left(a^{2}+b^{2}\right)(-a N+b M) \boldsymbol{\mu}\right)(t, \lambda) .
\end{aligned}
$$

By a direct calculation, we have the curvature $(\widetilde{m}, \widetilde{n}, \widetilde{L}, \widetilde{M}, \widetilde{N})$ of $(\widetilde{\gamma}, \widetilde{\nu})$.

Proposition 5.6. Under the same assumptions in Proposition 5.5, suppose that $e: U \rightarrow I \times \Lambda$ is a pre-envelope of $\left(\gamma, \nu_{1}, \nu_{2}\right)$ and $E_{\gamma}: U \rightarrow \mathbb{R}^{3}$ is an envelope. Then $e: U \rightarrow I \times \Lambda$ is also a pre-envelope of $(\widetilde{\gamma}, \widetilde{\nu}): I \times \Lambda \rightarrow \Delta_{2}$. Moreover, we have $E_{\widetilde{\gamma}}(u)=\widetilde{E}_{\gamma}(u)$ for all $u \in U$, where $E_{\widetilde{\gamma}}(u)=\widetilde{\gamma} \circ e(u)$ and $\widetilde{E}_{\gamma}(u)=$ $E_{\gamma}(u) /\left|E_{\gamma}(u)\right|$.

Proof. Since $\left(\gamma, \nu_{1}, \nu_{2}\right): I \times \Lambda \rightarrow \mathbb{R}^{3} \times \Delta_{2}$ is a one-parameter family of framed curves and $e: U \rightarrow I \times \Lambda$ is a pre-envelope of $\left(\gamma, \nu_{1}, \nu_{2}\right)$, we have $\gamma_{\lambda}(e(u))$. $\nu_{1}(e(u))=0$ and $\gamma_{\lambda}(e(u)) \cdot \nu_{2}(e(u))=0$ for all $u \in U$. It follows that

$$
\begin{aligned}
\widetilde{\gamma}_{\lambda}(e(u)) \cdot \widetilde{\nu}(e(u)) & =\left(\frac{\gamma_{\lambda}|\gamma|-\gamma|\gamma|_{\lambda}}{|\gamma|^{2}} \cdot \widetilde{\nu}\right) \circ(e(u))=\left(\frac{\gamma_{\lambda}}{|\gamma|} \cdot \widetilde{\nu}-\frac{|\gamma| \lambda}{|\gamma|} \widetilde{\gamma} \cdot \widetilde{\nu}\right) \circ(e(u)) \\
& =\frac{\gamma_{\lambda} \cdot\left(b \nu_{1}-a \nu_{2}\right)}{|\gamma| \sqrt{a^{2}+b^{2}}} \circ(e(u))=0 .
\end{aligned}
$$

Therefore $e: U \rightarrow I \times \Lambda$ is also a pre-envelope of $(\widetilde{\gamma}, \widetilde{\nu})$. Moreover, $E_{\widetilde{\gamma}}(u)=$ $\widetilde{\gamma}(e(u))=\widetilde{\gamma(e(u))}=\widetilde{E}_{\gamma}(u)$. Thus, we have $E_{\widetilde{\gamma}}(u)=\widetilde{E}_{\gamma}(\mathrm{u})$ for all $u \in U$.

Conversely, we have the following result.

Proposition 5.7. Let $(\gamma, \nu): I \times \Lambda \rightarrow \Delta_{2}$ be a one-parameter family of spherical Legendre curves with the curvature $(m, n, L, M, N)$. Then $(\gamma, \gamma, \nu): I \times \Lambda \rightarrow$ $S^{2} \times \Delta_{2} \subset \mathbb{R}^{3} \times \Delta_{2}$ is a one-parameter family of framed curves with the curvature $(\ell, m, n, \alpha, L, M, N, P, Q, R)=(0, m, n, m, L, M, N, 0, L, M)$.

Proof. Since $(\gamma, \nu): I \times \Lambda \rightarrow \Delta_{2}$ is a one-parameter family of spherical Legendre curves, we have $\gamma(t, \lambda) \cdot \nu(t, \lambda)=0$ and $\gamma_{t}(t, \lambda) \cdot \nu(t, \lambda)=0$ for all $(t, \lambda) \in I \times \Lambda$. Therefore, $(\gamma, \gamma, \nu): I \times \Lambda \rightarrow S^{2} \times \Delta_{2} \subset \mathbb{R}^{3} \times \Delta_{2}$ is a oneparameter family of framed curves. By a direct calculation, we have the curvature of $(\gamma, \gamma, \nu)$.

Proposition 5.8. Under the same notations in Proposition 5.7, suppose that $e: U \rightarrow I \times \Lambda$ is a pre-envelope of $(\gamma, \nu)$ and $E_{\gamma}: U \rightarrow S^{2}$ is an envelope. Then 
$e: U \rightarrow I \times \Lambda$ is also a pre-envelope of $(\gamma, \gamma, \nu): I \times \Lambda \rightarrow S^{2} \times \Delta_{2} \subset \mathbb{R}^{3} \times \Delta_{2}$. Moreover, $E_{\gamma}$ is also an envelope of $(\gamma, \gamma, \nu)$.

Proof. Since $\gamma_{\lambda}(e(u)) \cdot \nu(e(u))=0$ for all $u \in U, e: U \rightarrow I \times \Lambda$ is also a pre-envelope of $(\gamma, \gamma, \nu)$. Therefore, $E_{\gamma}$ is also an envelope of $(\gamma, \gamma, \nu)$.

\section{Examples}

Example 6.1. Let $n_{1}, n_{2}, n_{3}, k_{1}$ and $k_{2}$ be natural numbers with $n_{2}=n_{1}+k_{1}$ and $n_{3}=n_{2}+k_{2}$. Let $\left(\gamma, \nu_{1}, \nu_{2}\right): \mathbb{R} \times \mathbb{R} \rightarrow \mathbb{R}^{3} \times \Delta_{2}$ be

$$
\begin{aligned}
\gamma(t, \lambda) & =\left(\frac{1}{n_{1}} t^{n_{1}}+\lambda, \frac{1}{n_{2}} t^{n_{2}}, \frac{1}{n_{3}} t^{n_{3}}\right), \nu_{1}(t, \lambda)=\frac{\left(-t^{k_{1}}, 1,0\right)}{\sqrt{1+t^{2 k_{1}}}}, \nu_{2}(t, \lambda) \\
& =\frac{\left(-t^{k_{1}+k_{2}},-t^{2 k_{1}+k_{2}}, 1+t^{2 k_{1}}\right)}{\sqrt{\left(1+t^{2 k_{1}}\right)\left(1+t^{2 k_{1}+2 k_{2}}\right)}} .
\end{aligned}
$$

Then $\left(\gamma, \nu_{1}, \nu_{2}\right)$ is a one-parameter family of framed curves. By a direct calculation, we have

$$
\boldsymbol{\mu}(t, \lambda)=\frac{1}{\sqrt{1+t^{2 k_{1}}+t^{2 k_{1}+2 k_{2}}}}\left(1, t^{k_{1}}, t^{k_{1}+k_{2}}\right) .
$$

Then the curvature is given by

$$
\begin{aligned}
& \ell(t, \lambda)=\frac{k_{1} t^{2 k_{1}+k_{2}-1}}{\left(1+t^{2 k_{1}}\right) \sqrt{1+t^{2 k_{1}}+t^{2 k_{1}+2 k_{2}}}}, \\
& m(t, \lambda)=\frac{-k_{1} t^{k_{1}-1}}{\sqrt{\left(1+t^{2 k_{1}}\right)\left(1+t^{2 k_{1}}+t^{2 k_{1}+2 k_{2}}\right)}}, \\
& n(t, \lambda)=-\frac{t^{k_{1}+k_{2}-1}\left(k_{1}+k_{2}+k_{2} t^{2 k_{1}}\right)}{\sqrt{1+t^{2 k_{1}}}\left(1+t^{2 k_{1}}+t^{2 k_{1}+2 k_{2}}\right)}, \\
& \alpha(t, \lambda)=t^{n_{1}-1} \sqrt{1+t^{2 k_{1}}+t^{2 k_{1}+2 k_{2}}}, \\
& L(t, \lambda)=M(t, \lambda)=N(t, \lambda)=0, \\
& P(t, \lambda)=-\frac{t^{k_{1}}}{1+t^{2 k_{1}}}, Q(t, \lambda)=-\frac{t^{k_{1}+k_{2}}}{\sqrt{\left(1+t^{2 k_{1}}\right)\left(1+t^{2 k_{1}+2 k_{2}}\right)}}, \\
& R(t, \lambda)=\frac{1}{\sqrt{1+t^{2 k_{1}}+t^{2 k_{1}+2 k_{2}}}} .
\end{aligned}
$$

If we take $e: \mathbb{R} \rightarrow \mathbb{R} \times \mathbb{R}, e(u)=(0, u)$, then the variability condition holds and we have $P(e(u))=Q(e(u))=0$ for all $u \in \mathbb{R}$. Therefore $e$ is a pre-envelope and an envelope $E_{\gamma}: \mathbb{R} \rightarrow \mathbb{R}^{3}$ is given by $E_{\gamma}(u)=(u, 0,0)$. 


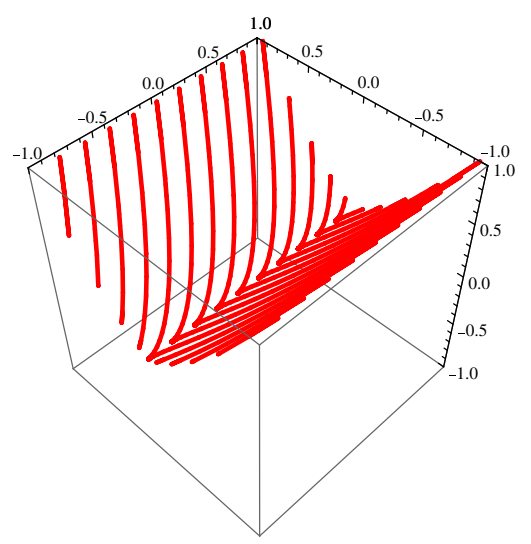

FIGURE $1 \gamma$

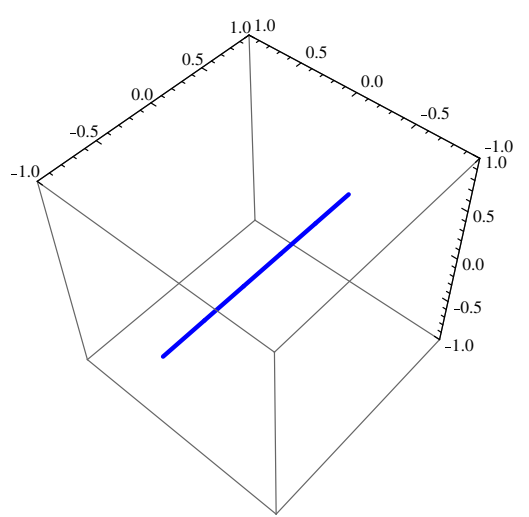

Figure $2 E_{\gamma}$

If we take $\left(n_{1}, n_{2}, n_{3}\right)=(2,3,4)$, we have a one-parameter family of framed curves

$$
\begin{aligned}
\gamma(t, \lambda) & =\left(\frac{1}{2} t^{2}+\lambda, \frac{1}{3} t^{3}, \frac{1}{4} t^{4}\right), \nu_{1}(t, \lambda)=\frac{(-t, 1,0)}{1+t^{2}}, \\
\nu_{2}(t, \lambda) & =\frac{\left(-t^{2},-t^{3}, 1+t^{2}\right)}{\sqrt{\left(1+t^{2}\right)\left(1+t^{2}+t^{4}\right)}} .
\end{aligned}
$$

See Figs. 1, 2 and 3.

Example 6.2. Let $n_{1}, n_{2}, n_{3}, m_{1}, m_{2}, m_{3}, k_{1}, k_{2}, h_{1}$ and $h_{2}$ be natural numbers with $n_{2}=n_{1}+k_{1}, n_{3}=n_{2}+k_{2}, m_{2}=m_{1}+h_{1}, m_{3}=m_{2}+h_{2}, h_{1} k_{2}=h_{2} k_{1}$ and $h_{1}$ or $k_{1}$ is 1 , or $h_{1}$ and $k_{1}$ are relatively prime. Let $\left(\gamma, \nu_{1}, \nu_{2}\right): \mathbb{R} \times \mathbb{R} \rightarrow \mathbb{R}^{3} \times \Delta_{2}$ 


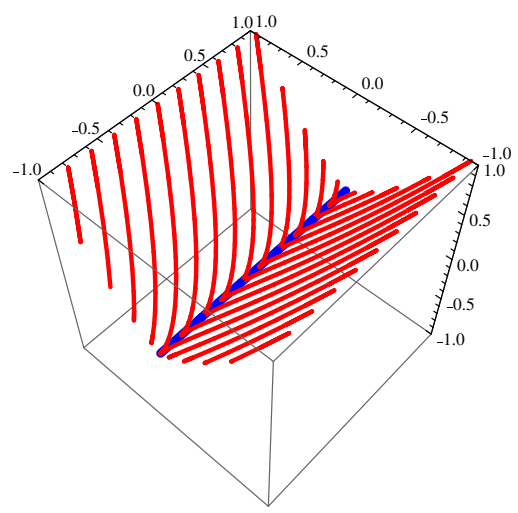

FiguRE $3 \gamma$ and $E_{\gamma}$

be

$$
\begin{aligned}
\gamma(t, \lambda) & =\left(\frac{t^{n_{1}}}{n_{1}}+\frac{\lambda^{m_{1}}}{m_{1}}, \frac{t^{n_{2}}}{n_{2}}+\frac{\lambda^{m_{2}}}{m_{2}}, \frac{t^{n_{3}}}{n_{3}}+\frac{\lambda^{m_{3}}}{m_{3}}\right), \nu_{1}(t, \lambda)=\frac{\left(-t^{k_{1}}, 1,0\right)}{\sqrt{1+t^{2 k_{1}}}}, \nu_{2}(t, \lambda) \\
& =\frac{\left(-t^{k_{1}+k_{2}},-t^{2 k_{1}+k_{2}}, 1+t^{2 k_{1}}\right)}{\sqrt{\left(1+t^{2 k_{1}}\right)\left(1+t^{2 k_{1}+2 k_{2}}\right)}} .
\end{aligned}
$$

Then $\left(\gamma, \nu_{1}, \nu_{2}\right)$ is a one-parameter family of framed curves. Moreover, by $\gamma_{\lambda}(t, \lambda)=\left(\lambda^{m_{1}-1}, \lambda^{m_{2}-1}, \lambda^{m_{3}-1}\right)$,

$$
\begin{aligned}
\gamma_{\lambda}(t, \lambda) \cdot \nu_{1}(t, \lambda)= & \frac{\lambda^{m_{1}-1}}{\sqrt{1+t^{2 k_{1}}}}\left(-t^{k_{1}}+\lambda^{h_{1}}\right), \\
\gamma_{\lambda}(t, \lambda) \cdot \nu_{2}(t, \lambda)= & \frac{\lambda^{m_{1}-1}}{\sqrt{\left(1+t^{2 k_{1}}\right)\left(1+t^{\left.2 k_{1}+2 k_{2}\right)}\right.}} \\
& \times\left(-t^{k_{1}+k_{2}}-t^{2 k_{1}+k_{2}} \lambda^{h_{1}}+\left(1+t^{2 k_{1}}\right) \lambda^{h_{1}+h_{2}}\right) .
\end{aligned}
$$

If we take $e: \mathbb{R} \rightarrow \mathbb{R} \times \mathbb{R}, e(u)=\left(u^{h_{1}}, u^{k_{1}}\right)$, then the variability condition holds, $\gamma_{\lambda}(e(u)) \cdot \nu_{1}(e(u))=0$ and $\gamma_{\lambda}(e(u)) \cdot \nu_{2}(e(u))=0$ for all $u \in \mathbb{R}$. Therefore $e$ is a pre-envelope and an envelope $E_{\gamma}: \mathbb{R} \rightarrow \mathbb{R}^{3}$ is given by

$$
E_{\gamma}(u)=\left(\frac{u^{h_{1} n_{1}}}{n_{1}}+\frac{u^{k_{1} m_{1}}}{m_{1}}, \frac{u^{h_{1} n_{2}}}{n_{2}}+\frac{u^{k_{1} m_{2}}}{m_{2}}, \frac{u^{h_{1} n_{3}}}{n_{3}}+\frac{u^{k_{1} m_{3}}}{m_{3}}\right) .
$$

If we take $\left(n_{1}, n_{2}, n_{3}, m_{1}, m_{2}, m_{3}\right)=(2,3,4,2,3,4)$, then $\gamma(t, \lambda)=\left(t^{2} / 2+\right.$ $\left.\lambda^{2} / 2, t^{3} / 3+\lambda^{3} / 3, t^{4} / 4+\lambda^{4} / 4\right)$ and an envelope $E_{\gamma}(u)=\left(u^{2}, 2 u^{3} / 3, u^{4} / 2\right)$, see Figs. 4, 5 and 6 .

Example 6.3. Let $\left(\boldsymbol{p}, \nu_{p 1}, \nu_{p 2}\right):[0,2 \pi) \rightarrow \mathbb{R}^{3} \times \Delta_{2}$ be an astroid $\boldsymbol{p}(t)=\left(\cos ^{3} t-\right.$ $\left.1, \sin ^{3} t, \cos 2 t-1\right), \nu_{p 1}(t)=(-\sin t,-\cos t, 0), \nu_{p 2}(t)=1 / 5(-4 \cos t, 4 \sin t, 3)$ and $\left(\boldsymbol{q}, \nu_{q 1}, \nu_{q 2}\right):[0,2 \pi) \rightarrow \mathbb{R}^{3} \times \Delta_{2}$ be given by $\boldsymbol{q}(\lambda)=\left(\cos ^{3} \lambda,-\sin ^{3} \lambda, \cos 2 \lambda\right)$, $\nu_{q 1}(t)=(\sin \lambda,-\cos \lambda, 0), \nu_{q 2}(t)=1 / 5(-4 \cos \lambda,-4 \sin \lambda, 3)$. Then $\left(\boldsymbol{p}, \nu_{p 1}, \nu_{p 2}\right)$ and $\left(\boldsymbol{q}, \nu_{q 1}, \nu_{q 2}\right)$ are framed curves. 


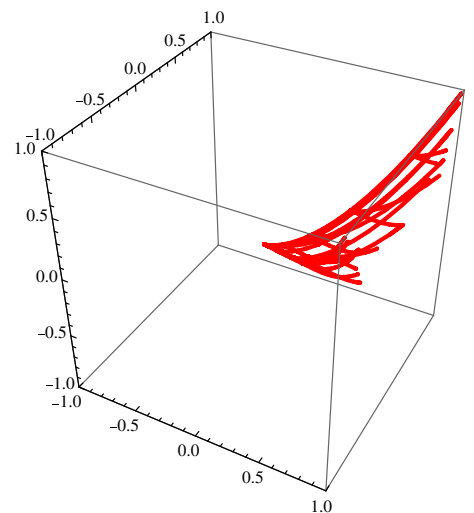

Figure $4 \gamma$

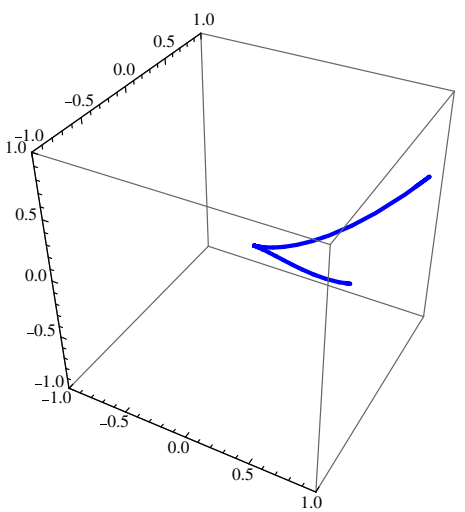

Figure $5 E_{\gamma}$

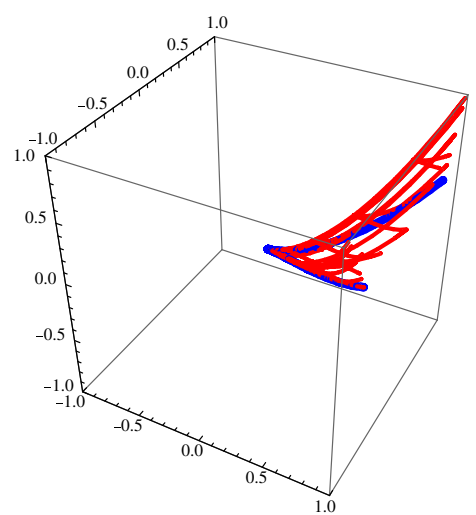

FiguRE $6 \gamma$ and $E_{\gamma}$ 


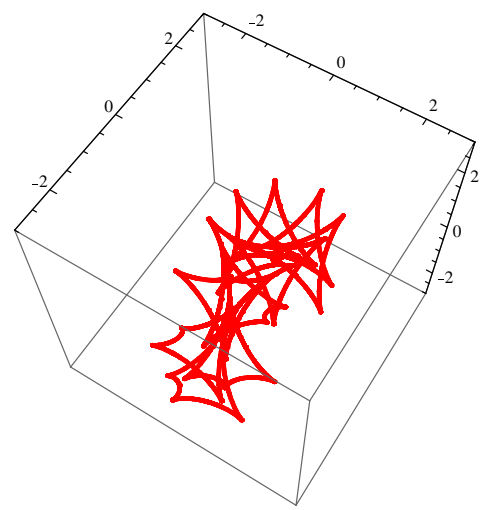

FiguRE $7 \gamma$

We consider a one-parameter family of framed curves $\left(\gamma, \nu_{1}, \nu_{2}\right):[0,2 \pi) \times$ $[0,2 \pi) \rightarrow \mathbb{R}^{3} \times \Delta_{2}$

$$
\gamma(t, \lambda)=\boldsymbol{q}(\lambda)+A(\lambda) \boldsymbol{p}(t), \nu_{1}(t, \lambda)=A(\lambda) \nu_{p 1}(t), \nu_{2}(t, \lambda)=A(\lambda) \nu_{p 2}(t),
$$

where

$$
A(\lambda)=\left(\begin{array}{ccc}
\cos \lambda & -\sin \lambda & 0 \\
\sin \lambda & \cos \lambda & 0 \\
0 & 0 & 1
\end{array}\right)
$$

that is

$$
\begin{aligned}
\gamma(t, \lambda) & =\left(\begin{array}{c}
\cos ^{3} \lambda \\
-\sin ^{3} \lambda \\
\cos 2 \lambda
\end{array}\right)+\left(\begin{array}{ccc}
\cos \lambda & -\sin \lambda & 0 \\
\sin \lambda & \cos \lambda & 0 \\
0 & 0 & 1
\end{array}\right)\left(\begin{array}{c}
\cos ^{3} t-1 \\
\sin ^{3} t \\
\cos 2 t-1
\end{array}\right), \\
\nu_{1}(t, \lambda) & =\left(\begin{array}{ccc}
\cos \lambda & -\sin \lambda & 0 \\
\sin \lambda & \cos \lambda & 0 \\
0 & 0 & 1
\end{array}\right)\left(\begin{array}{c}
-\sin t \\
-\cos t \\
0
\end{array}\right), \\
\nu_{2}(t, \lambda) & =1 / 5\left(\begin{array}{ccc}
\cos \lambda & -\sin \lambda & 0 \\
\sin \lambda & \cos \lambda & 0 \\
0 & 0 & 1
\end{array}\right)\left(\begin{array}{c}
-4 \cos t \\
4 \sin t \\
3
\end{array}\right) .
\end{aligned}
$$

By a direct calculation, we have

$$
\begin{aligned}
& \gamma_{\lambda}(t, \lambda) \cdot \nu_{1}(t, \lambda)=\sin t\left(15 \sin \lambda \cos \lambda-2 \cos ^{3} t-\cos t+3\right), \\
& \gamma_{\lambda}(t, \lambda) \cdot \nu_{2}(t, \lambda)=4(\cos t-1)(45 \sin \lambda \cos \lambda-2 \cos t-1) .
\end{aligned}
$$

If we take $e:[0,2 \pi) \rightarrow[0,2 \pi) \times[0,2 \pi), e(u)=(0, u)$, then $\gamma_{\lambda}(e(u)) \cdot \nu_{1}(e(u))=$ 0 and $\gamma_{\lambda}(e(u)) \cdot \nu_{2}(e(u))=0$ for all $u \in[0,2 \pi)$. Therefore $e$ is a pre-envelope and an envelope $E_{\gamma}:[0,2 \pi) \rightarrow \mathbb{R}^{3}$ is given by $E_{\gamma}(u)=\boldsymbol{q}(u)=\left(\cos ^{3} u,-\sin ^{3} u\right.$, $\cos 2 u$ ), see Figs. 7, 8 and 9 . 


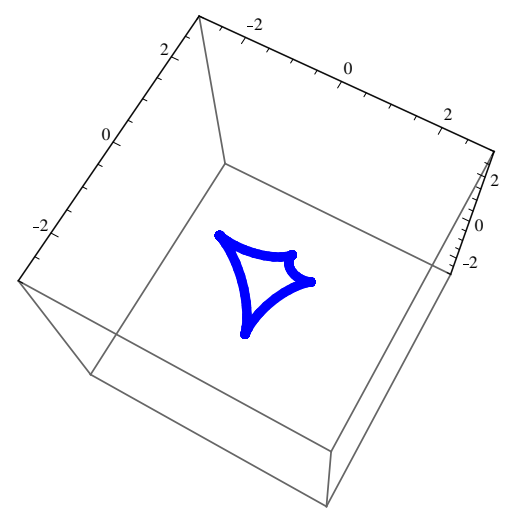

FigurE $8 E_{\gamma}$

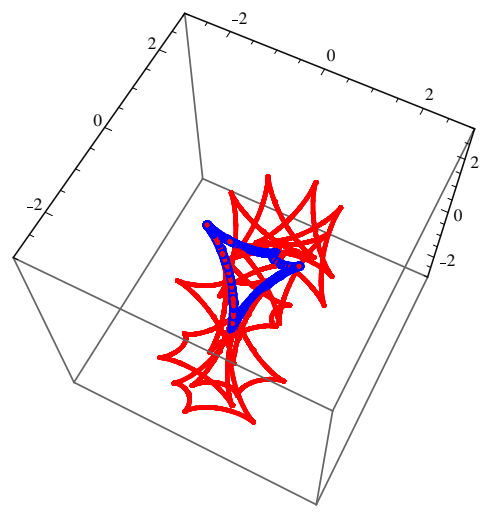

Figure $9 \gamma$ and $E_{\gamma}$

Example 6.4. Let $\left(\gamma, \nu_{1}, \nu_{2}\right): \mathbb{R} \times[0,2 \pi) \rightarrow \mathbb{R}^{3} \times \Delta_{2}$ be

$$
\begin{aligned}
\gamma(t, \theta)= & (\cos \theta(t \sin t+\cos t)-\sin \theta(-t \cos t+\sin t)+\theta \sin \theta+\cos \theta, \\
& \left.\sin \theta(t \sin t+\cos t)+\cos \theta(-t \cos t+\sin t)-\theta \cos \theta+\sin \theta, \frac{1}{2} t^{2}+\frac{1}{2} \theta^{2}\right), \\
\nu_{1}(t, \theta)= & (\cos \theta \sin t+\sin \theta \cos t, \sin \theta \sin t-\cos \theta \cos t, 0), \\
\nu_{2}(t, \theta)= & \frac{1}{\sqrt{2}}(\cos \theta \cos t-\sin \theta \sin t, \sin \theta \cos t+\cos \theta \sin t,-1) .
\end{aligned}
$$

Then $\left(\gamma, \nu_{1}, \nu_{2}\right)$ is a one-parameter family of framed curves. By a direct calculation, we have

$$
\begin{aligned}
& \gamma_{\theta}(t, \theta) \cdot \nu_{1}(t, \theta)=\sin t(t \cos t-\sin t+\theta), \\
& \gamma_{\theta}(t, \theta) \cdot \nu_{2}(t, \theta)=\frac{1}{\sqrt{2}}\left(t \cos ^{2} t-\sin t \cos t+\theta \cos t-\theta\right) .
\end{aligned}
$$




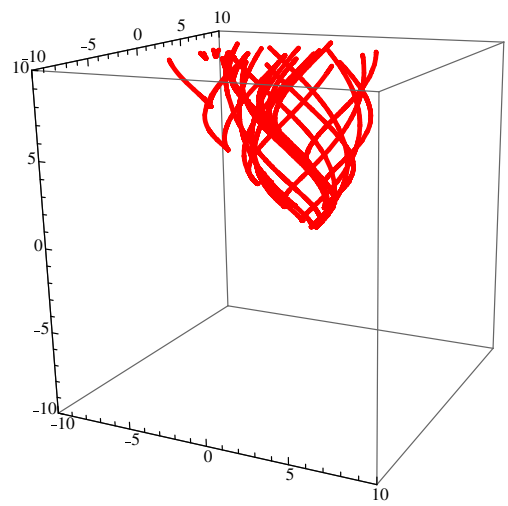

FiguRE $10 \gamma$

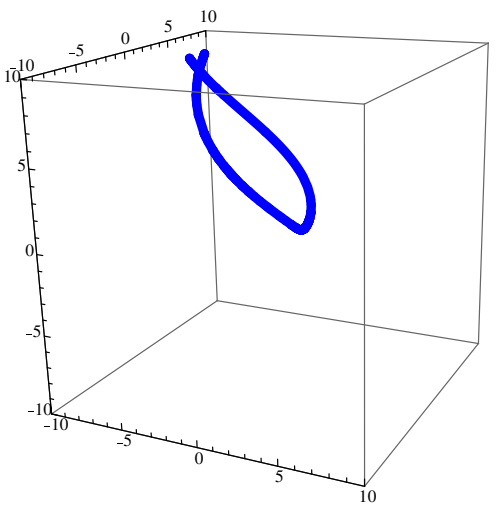

FIGURE $11 E_{\gamma}$

If we take $e: \mathbb{R} \rightarrow \mathbb{R} \times[0,2 \pi), e(u)=(0, u)$, we have $\gamma_{\theta}(e(u)) \cdot \nu_{1}(e(u))=0$ and $\gamma_{\theta}(e(u)) \cdot \nu_{2}(e(u))=0$ for all $u \in \mathbb{R}$. Therefore $e$ is a pre-envelope and an envelope $E_{\gamma}: \mathbb{R} \rightarrow \mathbb{R}^{3}$ is given by

$$
E_{\gamma}(u)=\left(u \sin u+2 \cos u,-u \cos u+2 \sin u, \frac{1}{2} u^{2}\right),
$$

see Figs. 10, 11 and 12.

Since $|\gamma(t, \theta)| \neq 0$, for all $(t, \theta) \in \mathbb{R} \times[0,2 \pi)$, we can consider

$$
\begin{aligned}
\widetilde{\gamma}(t, \theta) & =\frac{\gamma(t, \theta)}{|\gamma(t, \theta)|} \\
& =\left(\frac{\cos \theta(t \sin t+\cos t)-\sin \theta(-t \cos t+\sin t)+\theta \sin \theta+\cos \theta}{\sqrt{\frac{1}{4} t^{4}+\frac{1}{4} \theta^{4}+t^{2}+\theta^{2}+\frac{1}{2} t^{2} \theta^{2}+2(t \sin t+\cos t)-2 \theta(-t \cos t+\sin t)+2}},\right.
\end{aligned}
$$




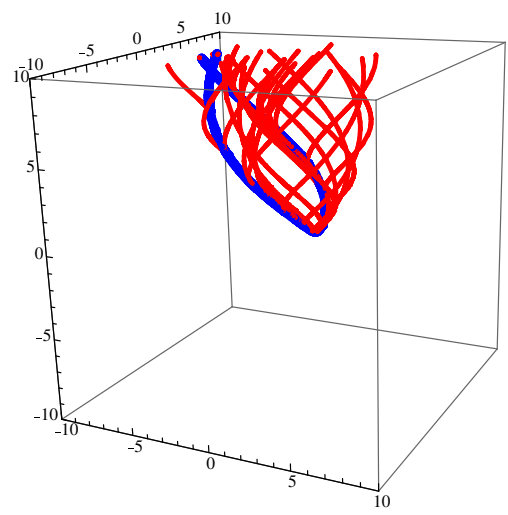

FIGURE $12 \gamma$ and $E_{\gamma}$

$$
\begin{aligned}
& \times \frac{\sin \theta(t \sin t+\cos t)+\cos \theta(-t \cos t+\sin t)-\theta \cos \theta+\sin \theta}{\sqrt{\frac{1}{4} t^{4}+\frac{1}{4} \theta^{4}+t^{2}+\theta^{2}+\frac{1}{2} t^{2} \theta^{2}+2(t \sin t+\cos t)-2 \theta(-t \cos t+\sin t)+2}}, \\
& \left.\times \frac{t^{2}+\theta^{2}}{2 \sqrt{\frac{1}{4} t^{4}+\frac{1}{4} \theta^{4}+t^{2}+\theta^{2}+\frac{1}{2} t^{2} \theta^{2}+2(t \sin t+\cos t)-2 \theta(-t \cos t+\sin t)+2}}\right) .
\end{aligned}
$$

Moreover, we have $\widetilde{\gamma}(t, \theta)=a(t, \theta) \nu_{1}(t, \theta)+b(t, \theta) \nu_{2}(t, \theta)+c(t, \theta) \boldsymbol{\mu}(t, \theta)$, where

$$
\begin{aligned}
& a(t, \theta)=\frac{t+\theta \cos t+\sin t}{\sqrt{\frac{1}{4} t^{4}+\frac{1}{4} \theta^{4}+t^{2}+\theta^{2}+\frac{1}{2} t^{2} \theta^{2}+2(t \sin t+\cos t)-2 \theta(-t \cos t+\sin t)+2}}, \\
& b(t, \theta)=\frac{1-\theta \sin t+\cos t-\frac{1}{2} t^{2}-\frac{1}{2} \theta^{2}}{\sqrt{2\left(\frac{1}{4} t^{4}+\frac{1}{4} \theta^{4}+t^{2}+\theta^{2}+\frac{1}{2} t^{2} \theta^{2}+2(t \sin t+\cos t)-2 \theta(-t \cos t+\sin t)+2\right)}}, \\
& c(t, \theta)=\frac{1-\theta \sin t+\cos t+\frac{1}{2} t^{2}+\frac{1}{2} \theta^{2}}{\sqrt{2\left(\frac{1}{4} t^{4}+\frac{1}{4} \theta^{4}+t^{2}+\theta^{2}+\frac{1}{2} t^{2} \theta^{2}+2(t \sin t+\cos t)-2 \theta(-t \cos t+\sin t)+2\right)}} .
\end{aligned}
$$

Then $a^{2}(t, \theta)+b^{2}(t, \theta) \neq 0$ for all $(t, \theta) \in \mathbb{R} \times[0,2 \pi)$ and we have $\widetilde{\nu}(t, \theta)=$ $(\widetilde{\gamma} \times \boldsymbol{\mu} /|\widetilde{\gamma} \times \boldsymbol{\mu}|)(t, \theta)$. By Proposition 5.5, $(\widetilde{\gamma}, \widetilde{\nu}): \mathbb{R} \times[0,2 \pi) \rightarrow \Delta_{2}$ is a oneparameter family of spherical Legendre curves. By Proposition 5.6, e : $\mathbb{R} \rightarrow$ $\mathbb{R} \times[0,2 \pi), e(u)=(0, u)$ is also a pre-envelope of $(\widetilde{\gamma}, \widetilde{\nu})$. By a direct calculation, we have

$$
E_{\widetilde{\gamma}}(u)=\widetilde{E}_{\gamma}(u)=\left(\frac{u \sin u+2 \cos u}{\sqrt{\frac{1}{4} u^{4}+u^{2}+4}}, \frac{-u \cos u+2 \sin u}{\sqrt{\frac{1}{4} u^{4}+u^{2}+4}}, \frac{u^{2}}{2 \sqrt{\frac{1}{4} u^{4}+u^{2}+4}}\right),
$$

see Figs. 13, 14 and 15. 


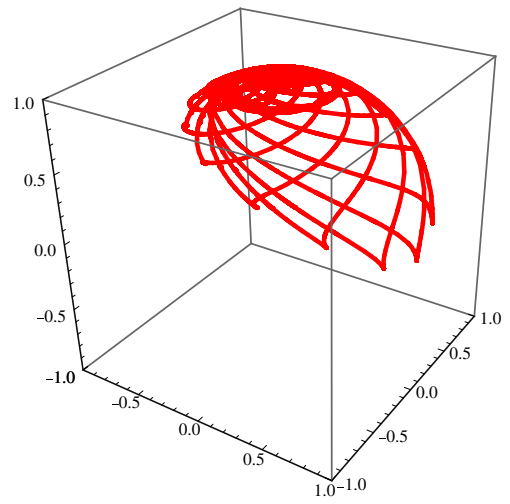

FigURE $13 \widetilde{\gamma}$

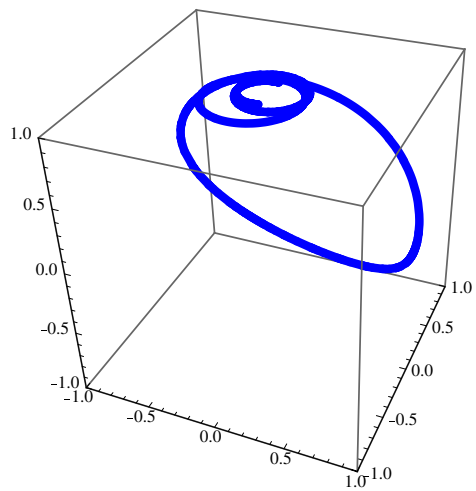

Figure $14 E_{\widetilde{\gamma}}$

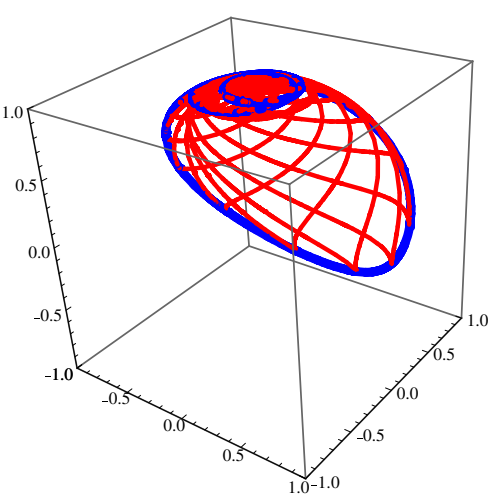

Figure $15 \widetilde{\gamma}$ and $E_{\widetilde{\gamma}}$ 


\section{Acknowledgements}

The authors would like to thank the referee for helpful comments to improve the original manuscript. The first author was supported by the National Natural Science Foundation of China (Grant No. 11671070) and the second author was supported by the JSPS KAKENHI (Grant No. JP 17K05238).

\section{Compliance with ethical standards}

Conflict of interest The authors declare that there is no conflict of interest in this work.

Open Access. This article is distributed under the terms of the Creative Commons Attribution 4.0 International License (http://creativecommons.org/licenses/ by/4.0/), which permits unrestricted use, distribution, and reproduction in any medium, provided you give appropriate credit to the original author(s) and the source, provide a link to the Creative Commons license, and indicate if changes were made.

Publisher's Note Springer Nature remains neutral with regard to jurisdictional claims in published maps and institutional affiliations.

\section{References}

[1] Bruce, J.W., Giblin, P.J.: What is an envelope? Math. Gaz. 65, 186-192 (1981)

[2] Bruce, J.W., Giblin, P.J.: Curves and Singularities. A Geometrical Introduction to Singularity Theory, 2nd edn. Cambridge University Press, Cambridge (1992)

[3] Carneiro, M.J.D.: Singularities of envelopes of families of submanifolds in $\mathbb{R}^{n}$. Ann. Sci. Ecole Norm. Sup. 16, 173-192 (1983)

[4] Fukunaga, T., Takahashi, M.: Framed surfaces in the Euclidean space. Bull. Braz. Math. Soc. New Ser. (2018). https://doi.org/10.1007/s00574-018-0090-z

[5] Fukunaga, T., Takahashi, M.: Framed surfaces and one-parameter families of framed curves. Preprint (2018)

[6] Gibson, C.G.: Elementary Geometry of Differentiable Curves. An Undergraduate Introduction. Cambridge University Press, Cambridge (2001)

[7] Gray, A., Abbena, E., Salamon, S.: Modern Differential Geometry of Curves and Surfaces with Mathematica. Studies in Advanced Mathematics, 3rd edn. Chapman and Hall/CRC, Boca Raton (2006)

[8] Honda, S., Takahashi, M.: Framed curves in the Euclidean space. Adv. Geom. 16, 265-276 (2016). https://doi.org/10.1515/advgeom-2015-0035

[9] Honda, S., Takahashi, M.: Evolutes and focal surface of framed immersions in the Euclidean space. Proc. R. Soc. Edinb. Sect. A Math. (2019). https://doi.org/ $10.1017 /$ prm.2018.84

[10] Ishikawa, G.: Generic bifurcations of framed curves in a space form and their envelopes. Topol. Appl. 159, 492-500 (2012) 
[11] Li, Y.-L., Pei, D.-H., Takahashi, M., Yu, H.-O.: Envelopes of Legendre curves in the unit spherical bundle over the unit sphere. Q. J. Math. (2018). https://doi. org/10.1093/qmath/hax056

[12] Rutter, J.W.: Geometry of Curves. Chapman \& Hall/CRC Mathematics. Chapman \& Hall/CRC, Boca Raton (2000)

[13] Takahashi, M.: Legendre Curves in the Unit Spherical Bundle Over the Unit Sphere and Evolutes Real and Complex Singularities. Contemporary Mathematics, vol. 675, pp. 337-355. American Mathematical Society, Providence (2016)

[14] Takahashi, M.: Envelopes of Legendre curves in the unit tangent bundle over the Euclidean plane. Results Math. 71, 1473-1489 (2017). https://doi.org/10.1007/ s00025-016-0619-7

[15] Thom, R.: Sur la théorie des enveloppes. J. Math. Pures Appl. (9) 41, 177-192 (1962)

Donghe Pei

Northeast Normal University

Changchun 130024 Jilin

China

e-mail: peidh340@nenu.edu.cn

Masatomo Takahashi

Muroran Institute of Technology

Muroran 050-8585

Japan

e-mail: masatomo@mmm.muroran-it.ac.jp

Haiou Yu

Jilin University of Finance and Economics

Changchun 130117 Jilin

China

e-mail: yuho930@nenu.edu.cn

Received: March 11, 2019.

Revised: August 21, 2019. 